ARTICLE

Received 19 Aug 2016 | Accepted 16 Jan 2017 | Published 3 Mar 2017

DOl: $10.1038 /$ ncomms14656

OPEN

\title{
Activation of E-prostanoid 3 receptor in macrophages facilitates cardiac healing after myocardial infarction
}

Juan Tang1,2, Yujun Shen², Guilin Chen², Qiangyou Wan ${ }^{1}$, Kai Wang ${ }^{1}$, Jian Zhang ${ }^{1,2}$, Jing Qin ${ }^{3,4}$, Guizhu Liu1, Shengkai Zuo ${ }^{1}$, Bo Tao', Yu Yu¹, Junwen Wang ${ }^{3,5,6}$, Michael Lazarus ${ }^{7} \&$ Ying $\mathrm{Yu}^{1,2}$

Two distinct monocyte (Mo)/macrophage (Mp) subsets (Ly6 $\mathrm{C}^{\text {low }}$ and Ly6 $\mathrm{C}^{\text {high}}$ ) orchestrate cardiac recovery process following myocardial infarction (MI). Prostaglandin (PG) $E_{2}$ is involved in the Mo/Mp-mediated inflammatory response, however, the role of its receptors in Mos/Mps in cardiac healing remains to be determined. Here we show that pharmacological inhibition or gene ablation of the Ep3 receptor in mice suppresses accumulation of Ly6Clow Mos/Mps in infarcted hearts. Ep3 deletion in Mos/Mps markedly attenuates healing after MI by reducing neovascularization in peri-infarct zones. Ep3 deficiency diminishes CX3C chemokine receptor 1 (CX3CR1) expression and vascular endothelial growth factor (VEGF) secretion in Mos/Mps by suppressing TGF $\beta 1$ signalling and subsequently inhibits Ly6Clow Mos/Mps migration and angiogenesis. Targeted overexpression of Ep3 receptors in Mos/Mps improves wound healing by enhancing angiogenesis. Thus, the $\mathrm{PGE}_{2} / \mathrm{Ep} 3$ axis promotes cardiac healing after MI by activating reparative Ly $6 \mathrm{C}^{\text {low }} \mathrm{Mos} / \mathrm{Mps}$, indicating that Ep3 receptor activation may be a promising therapeutic target for acute $\mathrm{MI}$.

\footnotetext{
${ }^{1}$ Key Laboratory of Food Safety Research, CAS Center for Excellence in Molecular Cell Science, Institute for Nutritional Sciences, Shanghai Institutes for Biological Sciences, Chinese Academy of Sciences, University of Chinese Academy of Sciences, Shanghai 200031, China. ${ }^{2}$ Department of Pharmacology, School of Basic Medical Sciences, Tianjin Medical University, Tianjin 300070, China. ${ }^{3}$ Center for Genomic Sciences, LKS Faculty of Medicine, The University of Hong Kong, 5 Sassoon Road, Hong Kong, SAR 999077, China. ${ }^{4}$ School of Life Science, Chinese University of Hong Kong, Hong Kong, SAR 999077, China. ${ }^{5}$ Division of Biomedical Statistics and Informatics, Center for Individualized Medicine, Mayo Clinic, Scottsdale, Arizona 85259, USA. 6 Department of Biomedical Informatics, Arizona State University, Scottsdale, Arizona 85259, USA. ${ }^{7}$ International Institute for Integrative Sleep Medicine (WPI-IIIS), University of Tsukuba, Tsukuba City, Ibaraki 305-8575, Japan. Correspondence and requests for materials should be addressed to Y.Y. (email: yuying@sibs.ac.cn or yuying@tmu.edu.cn).
} 
$\mathrm{H}$ ealing of the infarcted myocardium involves a complex and coordinated process of inflammation, angiogenesis and tissue remodelling. Monocytes (Mos) and macrophages (Mps) in the infarcted myocardium are the essential immune cells for determining the progression and resolution of inflammation and repair following myocardial infarction (MI) ${ }^{1}$. Disruption of the Mo/Mp-mediated inflammatory response by $\mathrm{Mo} / \mathrm{Mp}$ depletion or inhibition of $\mathrm{Mo} / \mathrm{Mp}$ migration impairs wound healing and deteriorates left ventricular remodelling after $\mathrm{MI}^{2-4}$. In contrast, controlled activation of Mos/Mps ameliorates cardiac function and post MI remodelling by inducing $\mathrm{Mo} / \mathrm{Mp}$ infiltration and neovascularization ${ }^{5}$. Two distinct subpopulations of Mos/Mps are involved in recovery after $\mathrm{MI}$ in a sequential pattern. Early responding Mos/Mps (Ly6 C ${ }^{\text {high }}$ ) are predominant 1-3 days after MI and display phagocytic and pro-inflammatory properties highly expressing tumour necrosis factor (TNF) $\alpha$, interleukin (IL-6), myeloperoxidase, and cathepsins, whereas late-responding Mos/Mps (Ly6C ${ }^{\text {low }}$ ) appear 4-7 days after MI and exhibit anti-inflammatory characteristics to support tissue regeneration by secreting reparative cytokines, such as IL-10, transforming growth factor (TGF) $\beta 1$, and vascular endothelial growth factor $(\mathrm{VEGF})^{6}$. Injured hearts express a unique chemokine profile over time to coordinate Mo recruitment, and the sequential recruitment of Ly6 $\mathrm{C}^{\text {high }}$ and Ly6 $6 \mathrm{C}^{\text {low }}$ Mos is dependent on C-C chemokine receptor type 2 (CCR2) and CX3C chemokine receptor 1 (CX3CR1), respectively ${ }^{6}$. Moreover, Ly6C ${ }^{\text {high }}$ Mos can convert to Ly6C low Mos in circulation 7,8 and inflamed tissues including infarcted hearts 9,10 . Delayed transition of Ly6Chigh (M1-like) to Ly6C ${ }^{\text {low }}$ Mos/Mps (M2-like), such as in atherosclerotic or diabetic animals ${ }^{11,12}$, attenuates wound repair post MI. Thus, targeting certain Mos/Mps as a therapeutic strategy for infarct healing and repair continues to receive intensive attention $^{13}$.

Prostaglandin (PG) $E_{2}$ is a key lipid mediator in many pathophysiological processes, including inflammation and immune responses, and it elicits diverse functions by acting on its four different E-prostanoid receptors (Ep1-Ep4) in a paracrine and autocrine manner ${ }^{14}$. Microsomal $\mathrm{PGE}_{2}$ synthase-1 (mPGES-1), an inducible terminal isomerase for $\mathrm{PGE}_{2}$ biosynthesis, is the major source of $\mathrm{PGE}_{2}$ formed in vivo and during inflammatory responses ${ }^{15}$. Disabling $\mathrm{PGE}_{2}$ generation by mPGHS-1 deletion leads to increased infarct sizes and adverse left ventricular remodelling after MI in mice ${ }^{16}$. Interestingly, mice with mPGES-1 deletion in bone marrowderived myeloid cells alone displayssimilar cardiac phenotypes in global mPGES-1-deficient mice as subjected to coronary ligation ${ }^{17}$, strongly implicating Mos/Mps-derived $\mathrm{PGE}_{2}$ in wound healing after MI. However, the underlying mechanisms are largely unknown.

In this study, we investigated the role of $\mathrm{PGE}_{2}$ receptors on recruitment of $\mathrm{Mo} / \mathrm{Mp}$ subsets during inflammation and further explored effects of genetic deletion or overexpression of Ep3 receptor in Mos/Mps on cardiac healing in mice after acute MI. We found, disruption of Ep3 receptor in Mos/Mps resulted in augmented infarct size and reduced cardiac functions after MI through suppression of reparative Ly6C $C^{\text {low }}$ infiltration and its mediated angiogenesis in peri-infarct zones in mice. Moreover, Ep3 deficiency in Mos/Mps inhibited TGF $\beta 1$ signalling to directly suppress transcription of $C x 3 \mathrm{cr} 1$ and $V e g f$ genes, therefore, retarding Ly6C $C^{\text {low }}$ migration and neovascularization in the infarcted hearts. Controlled activation of Ep3 receptor in Mos/Mps facilitated Ly6 $\mathrm{C}^{\text {low }}$ infiltration and subsequent healing of MI by activation of TGF $\beta 1$ signalling. Thus, $\mathrm{PGE}_{2} / \mathrm{Ep} 3$ axis facilites cardiac healing after MI by activating reparative Ly6C ${ }^{\text {low }}$ Mos/Mps.

\section{Results}

Ep3 mediates recruitment of $\mathrm{Ly}^{6} \mathrm{C}^{\text {low }} \mathrm{Mos} / \mathrm{Mps}$ in peritonitis. To explore which $\mathrm{PGE}_{2}$ receptor subtype(s) mediate $\mathrm{Mo} / \mathrm{Mp}$ recruitment in zymosan-induced peritonitis in mice, both Ly6C $^{\text {high }}$ and Ly6C ${ }^{\text {low }}$ Mos/Mps were sorted by flow cytometry (Fig. 1a). Western blot analysis confirmed that the surface markers CCR2 and CX3CR1 were abundantly expressed in Ly6C $C^{\text {high }}$ and Ly6C ${ }^{\text {low }}$ cells, respectively (Supplementary Fig. 1a), and reverse transcriptase polymerase chain reaction (RT-PCR) showed that more pro-inflammatory genes were expressed in Ly6C $\mathrm{C}^{\text {high }}$ cells, while more reparative cytokines were expressed in Ly6C ${ }^{\text {low }}$ cells (Supplementary Fig. 1b,c). All $\mathrm{PGE}_{2}$ receptors (Ep1-4) were differentially expressed in both Ly6Chigh and Ly6 $\mathrm{C}^{\text {low }} \mathrm{Mos} / \mathrm{Mps}$, and Ly6C ${ }^{\text {low }}$ Mos/Mps expressed more Ep1 and Ep3 receptors than Ly6C ${ }^{\text {high }}$ Mos/Mps (Fig. 1b). Interestingly, the Ep3 receptor inhibitor L-798,106 reduced peritoneal infiltration of Ly6C ${ }^{\text {low }}$ and total Mos/Mps without significantly influencing Ly6C $\mathrm{C}^{\text {high }} \mathrm{Mos} / \mathrm{Mps}$, while the Ep1 receptor inhibitor SC-51322 retarded recruitment of Ly6C ${ }^{\text {high }}$ Mos/Mps $48 \mathrm{~h}$ after the zymosan challenge (Fig. 1c-e). Consistently, L-798,106 significantly suppressed expression of reparative and pro-angiogenic cytokines (IL-13, TGF $\beta 1, V E G F$ and MMP9) and CX3CR1 in infiltrated Mos/Mps (Fig. 1f) without markedly altering expression of pro-inflammatory cytokines (Fig. 1g). In addition, by using myeloid cell-specific Ep3-deficient mice $\left(E p 3^{F / F} ; L y s M^{C r e}\right.$, Fig. 2a), we also found Ep3 deficiency in Mos/Mps (Fig. 2b) markedly reduced total peritoneal Mos/Mps by restraining infiltration of Ly6C $\mathrm{C}^{\text {low }} \mathrm{Mos} / \mathrm{Mps}$ in response to a zymosan challenge in mice (Fig. 2c-e). Similarly, Ep3 deletion downregulated reparative cytokines and CX3CR1 gene expression in Mos/Mps without overt effects on the expression of the pro-inflammatory cytokines tested (Fig. 2f,g).

We then examined the effect of Ep3 deletion on the differentiation of recruited Mos in zymosan-induced peritonitis in mice. By day 1 after adoptive transfer (Supplementary Fig. 2a), $\approx 30 \%$ of the accumulated Ly6C $\mathrm{C}^{\text {high }}$ Mos had Ly6C $\mathrm{C}^{\text {low }}$ marker (Supplementary Fig. 2b), while Ep3 deficiency did not significantly influence Ly6C ${ }^{\text {high }}$ Mo infiltration (Supplementary Fig. 2c) and its differentiation toward Ly6 $\mathrm{C}^{\text {low }}$ Mos/Mps (Supplementary Fig. 2d). In contrast, deletion of Ep3 receptor markedly reduced infiltration of Ly6C $\mathrm{C}^{\text {low }} \mathrm{Mos} / \mathrm{Mps}$ in zymosan-challenged peritoneal cavity (Supplementary Fig. 2e,f). Taken together, these results suggest that the Ep3 receptor is involved in mediating the recruitment of Ly6 $\mathrm{C}^{\text {low }} \mathrm{Mos} / \mathrm{Mps}$ in response to inflammatory insults.

Ep3 promotes cardiac recovery by recruiting $\mathrm{Ly}_{6 \mathrm{C}} \mathrm{C}^{\mathrm{low}} \mathrm{Mo} / \mathrm{Mp}$. Two Mo/Mp subsets (Ly6Clow and Ly6 $\mathrm{C}^{\text {high }}$ ) are also implicated in recovery after $\mathrm{MI}-\mathrm{a}$ sterile inflammatory reaction ${ }^{6}$. We then examined the role of $\mathrm{Mo} / \mathrm{Mp}$ in cardiac repair after ischaemia in mice. As expected, all PG products, including $\mathrm{PGE}_{2}$, were elevated in infarcted hearts, although Ep3 deficiency in Mos/Mps had no significant influence on PG production (Supplementary Fig. 3a-e). CD11b ${ }^{+} \mathrm{Ly}_{6 \mathrm{G}}{ }^{-} \mathrm{CD} 115^{+}$Mos/Mps were sorted from infarcted hearts in mice (Fig. 3a). Notably, total infiltrated $\mathrm{Mos} / \mathrm{Mps}$ in hearts were significantly reduced in $E p 3^{F / F} ; L y s M^{C r e}$ mice starting from 4 days after left anterior descending (LAD) artery ligation compared with those in $\mathrm{EP}^{\mathrm{F} / \mathrm{F}}$ controls (Fig. 3b), through suppression of recruitment of $\mathrm{CD} 11 \mathrm{~b}^{+} \mathrm{Ly}_{6 \mathrm{G}}{ }^{-} \mathrm{CD} 115^{+}$ Ly6C ${ }^{\text {low }}$ Mos/Mps (Fig. 3c) not $\mathrm{CD}_{11 b^{+}} \mathrm{Ly}_{6 \mathrm{G}}{ }^{-} \mathrm{CD} 115^{+}$ Ly6C ${ }^{\text {high }}$ Mos/Mps (Fig. 3d), which was further confirmed by using additional F4/80 marker (Supplementary Fig. 4a-e). While myeloid-Ep3 deficiency had no significant effect on total residential Mps in spleens, lungs, livers and hearts (Supplementary Fig. $5 \mathrm{a}-\mathrm{d})$, and on circulating Mos and neutrophils in 

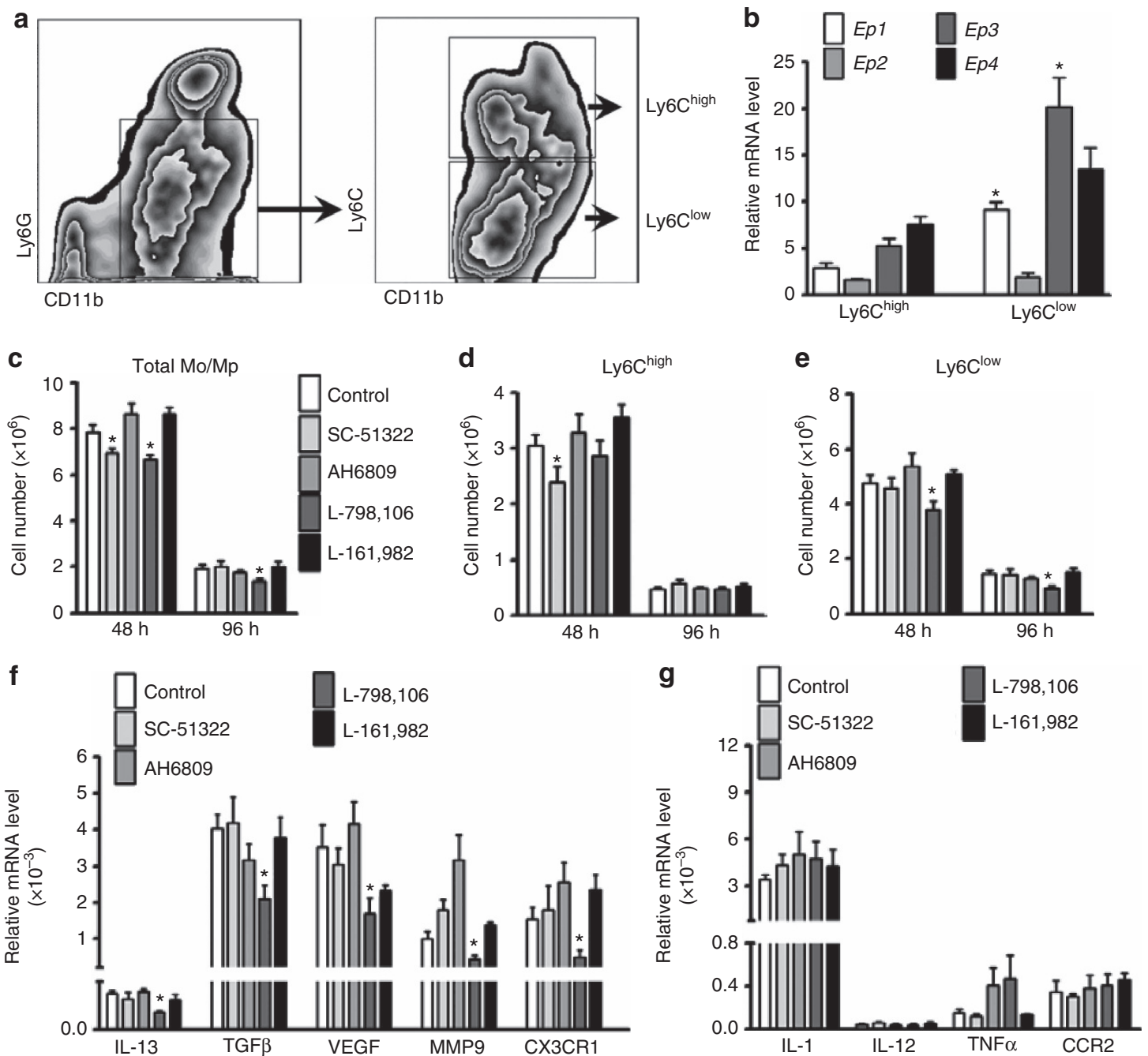

Figure 1 | Ep3 blockade represses recruitment of Ly6 $\mathbf{C}^{\text {low }}$ Mos/Mps in peritonitis in mice. (a) Gating strategy for peritoneal Ly6 high and Ly6 $\mathrm{C}^{\text {low }}$ Mos/Mps in zymosan-induced peritonitis in mice. (b) Relative mRNA levels of the PG $E_{2}$ receptors (Ep1-4) in peritoneal Ly6Chigh and Ly6Clow Mos/Mps; data represent mean \pm s.e.m. ${ }^{*} P<0.05$ versus $L y 6 C^{\text {high }}$ Mos/Mps (unpaired two-tailed $t$-test); $n=5$. (c-e) Effect of administration of different $P G E_{2}$ receptor blockers on recruitment of total Mos/Mps (c), Ly6Chigh (d), and Ly6Clow (e) Mos/Mps at $48 \mathrm{~h}$ and $96 \mathrm{~h}$ after a zymosan challenge in mice. Data represent mean \pm s.e.m. ${ }^{\star} P<0.05$ versus control(unpaired two-tailed $t$-test); $n=5-6$. SC-51322, Ep1 inhibitor; AH6809, Ep2 inhibitor; L-798106, Ep3 inhibitor; L-161982, Ep4 inhibitor. (f,g) Effect of different PGE 2 receptor blockers on expression of anti-inflammatory (f) and pro-inflammatory $(\mathbf{g})$ markers in peritoneal Mos/Mps collected $48 \mathrm{~h}$ after zymosan treatment. Data represent mean \pm s.e.m. ${ }^{\star} P<0.05$ versus control (unpaired two-tailed $t$-test); $n=4$.

mice either (Supplementary Fig. 6a,b). Again, Mo adoptive transfer confirmed Ep3 deficiency resulted in decreased Ly6C low infiltration without overt influence on Ly6Chigh differentiation in circulation and infracted hearts (Supplementary Fig. 7a-i). The Ly6C ${ }^{\text {low }} \mathrm{Mo} / \mathrm{Mp}$ surface marker CX3CR1 and VEGF expression in the infiltrated $\mathrm{Mos} / \mathrm{Mps}$ were reduced by half in $E p 3^{F / F} ; L y s M^{C r e}$ mice (Fig. 3e), and the CX3CR1 ligand CX3CL1 expression was not altered in hearts from $E p 3^{F / F} ; L y s M^{C r e}$ mice (Supplementary Fig. 8a-c). Moreover, Ep3 deletion did not influence proliferation and apoptosis of Mos/Mps infiltrated in infarcted hearts (Supplementary Fig. 9a-d). Immunostaining further confirmed reduction of VEGF expression in Mos/Mps in injured hearts in $E p 3^{F / F} ; L y s M^{C r e}$ mice (Fig. 3f,g). Accordingly, in $E p 3^{F / F} ; L y s M^{C r e}$ mice, neovascularization in the ischaemic zone at a late stage (day 14 after LAD ligation) was also diminished (Fig. 3h-j), infarct areas were expanded (Supplementary Fig. 10a,b), and cardiac function recovery was markedly impaired after MI (Fig. 3k,l, Supplementary Fig. 10c, Supplementary
Table 1). However, $E p 3^{F / F} ; L y s M^{C r e}$ mice had normal cardiac function at basal condition and even after dobutamine challenge (Supplementary Table 2), and Ep3 deficiency did not influence the number and functions of neutrophil infiltrated in the infarcted hearts (Supplementary Fig. 11a-h).

$\mathrm{Mps}$, as the major source of matrix metallopeptidases (MMPs) and TGF $\beta 1$, play an important role in cardiac remodelling and fibrosis $^{18}$. Mo/Mp-Ep3 deletion, indeed, caused less non-vascular smooth muscle actin (SMA) positive myofibroblasts (Supplementary Fig. 12a,b), downregulation of $M M P 9$, collagen I, III and Thrombospondin1 (THBS1) expression in infarcted hearts in mice (Supplementary Fig. 12c-j). Consistently, Masson's trichrome staining showed an increased myocardial scar size with decreased collagen deposition in $E p 3^{F / F} ; L y s M^{C r e}$ mice at both day 7 and 14 after MI (Supplementary Fig. 13a-c). We did not observe significant difference of early necrotic area between $E p 3^{F / F} ; L y s M^{C r e}$ and $E p 3^{F / F}$ mice after MI (Supplementary Fig. 13d,e). Taken together, myeloid-Ep3 deletion impairs 
a

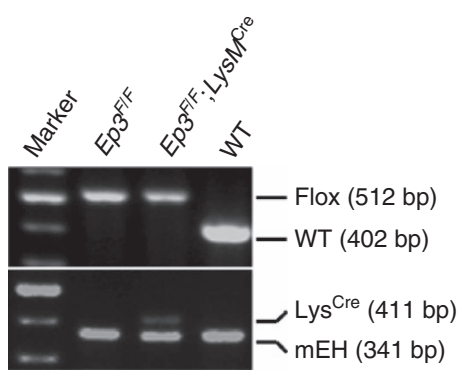

b
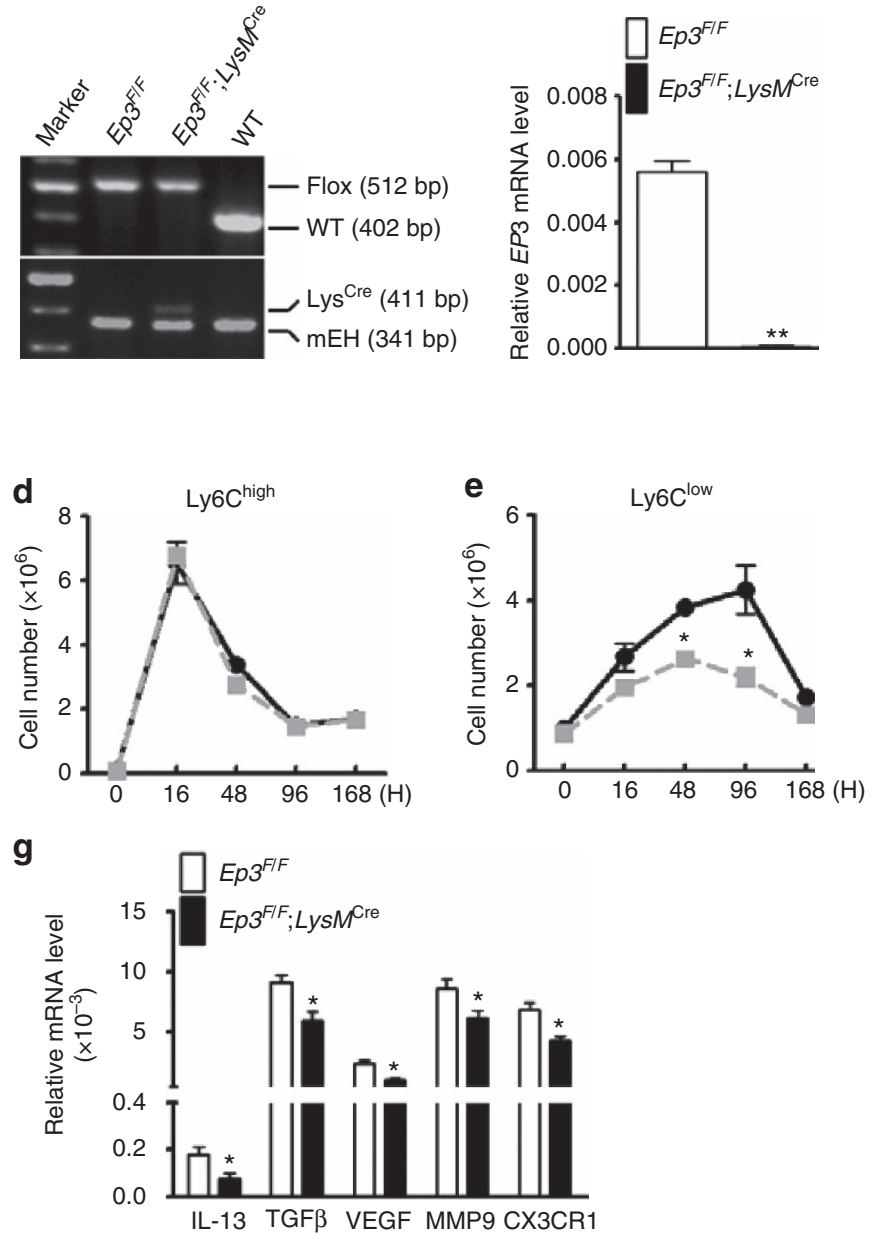

c

$E p 3^{F / F}$

$E p 3^{F / F} ; L y s M^{C r e}$
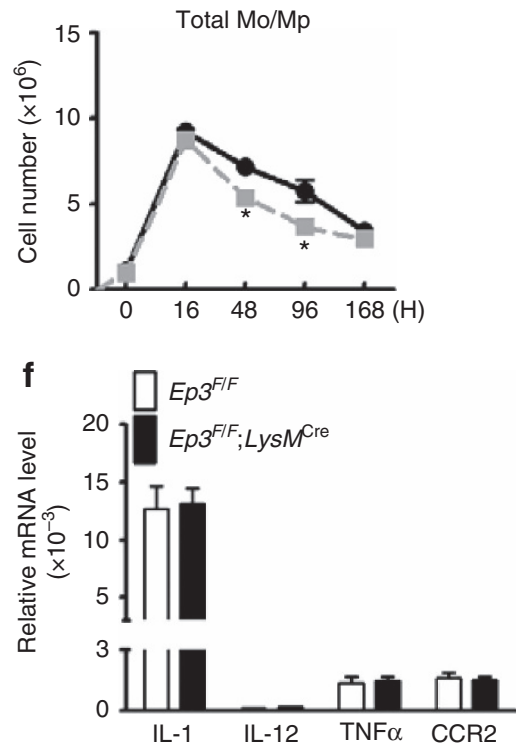

Figure 2 | Ep3 deletion suppresses Ly6 $\mathbf{C}^{\text {low }} \mathbf{M o} / \mathbf{M p}$ infiltration in peritonitis in mice. (a) Genotyping of Ep3 $3^{F / F} ; L y s M^{C r e}$ mice. Microsomal epoxide hydrolase $(\mathrm{mEH})$ was used as quality control for extracted DNA from mouse tail biopsy. (b) Ep3 receptor expression levels in peritoneal Mps from $E p 3^{F / F} ; L y s M^{C r e}$ and $E p 3^{F / F}$ mice. Data represent mean \pm s.e.m. ${ }^{\star \star} P<0.01$ versus $E p 3^{F / F}$ (unpaired two-tailed $t$-test); $n=10$. (c-e) Effect of Ep3 deletion on recruitment of total Mos/Mps (c), Ly6Chigh (d), and Ly6Clow subtype (e) Mos/Mps 48 and $96 \mathrm{~h}$ after a zymosan challenge in mice. Data represent mean \pm s.e.m. ${ }^{\star} P<0.05$ versus $E p 3^{F / F}$ (unpaired two-tailed $t$-test); $n=5-7$. (f/g) Effect of Ep3 deficiency on expression of proinflammatory $(\mathbf{f})$ and reparative angiogenic markers $(\mathbf{g})$ in peritoneal Mos/Mps collected $48 \mathrm{~h}$ after zymosan treatment. Data represent mean \pm s.e.m. ${ }^{\star} P<0.05$ versus Ep $3^{\mathrm{F} / F}$ (unpaired two-tailed $t$-test); $n=10-11$.

cardiac recovery from infarction by suppression of Ly6 $\mathrm{C}^{\text {low }} \mathrm{Mo} /$ Mp-mediated angiogenesis and cardiac fibrosis in mice (Supplementary Table 3).

Given that Ep3 deficiency inhibited VEGF expression in reparative Ly6C ${ }^{\text {low }} \mathrm{Mps}$, we tested the effect of the Mp Ep3 receptor on angiogenesis in vitro. Indeed, VEGF expression was diminished at both the mRNA (Supplementary Fig. 14a) and protein levels (Supplementary Fig. 14b,c) in cultured Mps from $E p 3^{F / F} ; L y s M^{C r e}$ mice. In a cultured three-dimensional angiogenesis model using HUVECs, their sprouting and tube structure formations were markedly reduced when co-cultured with peritoneal Mps from $E p 3^{F / F} ; L y s M^{C r e}$ mice compared to those in models co-cultured from $E p 3^{F / F}$ controls (Supplementary Fig. 14d-g).

To validate the role of Ep3 receptor in Mos/Mps, we examined whether bone marrow transplantation (BMT) from wild-type (WT) donors could rescue the defective function of Ly6Clow Mos/Mps in Ep3 knockout (KO) mice. Genotyping of both tail specimens and blood samples verified successful BM reconstitution (Fig. 4a). Notably, total Ly6C ${ }^{\text {low }}$, not Ly6C ${ }^{\text {high }}$ Mos/Mps recruited in ischaemic hearts were significantly reduced in WT mice which underwent BMT from Ep3 $\mathrm{KO}$ mice $\mathrm{BM}(\mathrm{KO} \rightarrow \mathrm{WT})$ (Fig. 4b-d). Moreover, the decreased expression of both VEGF and CX3CR1 in EP3 KO Ly6C ${ }^{\text {low }}$ Mos/Mps in the infarcted hearts was completely rectified by WT BMT (WT $\rightarrow \mathrm{KO}$, Fig. $4 \mathrm{e})$. In line with the recovered infiltration and function of Ly6C $\mathrm{C}^{\text {low }} \mathrm{Mos} / \mathrm{Mps}$, neovascularization in peri-infarct zones and cardiac function after MI were significantly improved in $\mathrm{WT} \rightarrow \mathrm{KO}$ mice as compared with that in $\mathrm{KO} \rightarrow \mathrm{KO}$ mice (Fig. $4 \mathrm{f}-\mathrm{i}$, Supplementary Table 4).

Ep3 deficiency inhibits CX3CR1 and VEGF expression in Mps. Reparative Ly6C ${ }^{\text {low }} \mathrm{Mo} / \mathrm{Mp}$ recruitment during inflammation, including that post $\mathrm{MI}$, depends on the CX3CR1 receptor ${ }^{6}$. We detected striking downregulation of CX3CR1 and VEGF expression in peritoneal Mps isolated from $E p 3^{F / F} ; L y s M^{C r e}$ mice challenged by zymosan at both $48 \mathrm{~h}$ (Fig. 5a, Supplementary Fig. 15a) and $96 \mathrm{~h}$ (Fig. 5b, Supplementary Fig. 15b) and in cultured Ep3-deficient Mps $\left(E p 3^{F / F} ; L y s M^{C r e}\right.$ mice) treated by $\mathrm{PGE}_{2}$ (Fig. 5c, Supplementary Fig. 15c). CCR2, the receptor for monocyte chemoattractant protein-1 (MCP-1) that mediates pro-inflammatory Ly6 $\mathrm{C}^{\text {high }} \mathrm{Mo} / \mathrm{Mp}$ recruitment, was not altered 
significantly in Ep3-deficient Mps (Fig. 5a-c). Accordingly, the migration of Ep3-deficient Mps in Boyden chambers was notably restrained in Ep3-deficient $\mathrm{Mps}$ with and without $\mathrm{PGE}_{2}$ stimulation (Fig. 5d).
Previously, we demonstrated that the Ep3 receptor mediates TGF $\beta 1$ signalling in pulmonary vascular smooth muscle cells by activation of Rho/ROCK ${ }^{19}$. Similarly, TGF $\beta 1$ signalling (phosphorylation of Smad2 and Smad3) was inhibited in Ep3- a
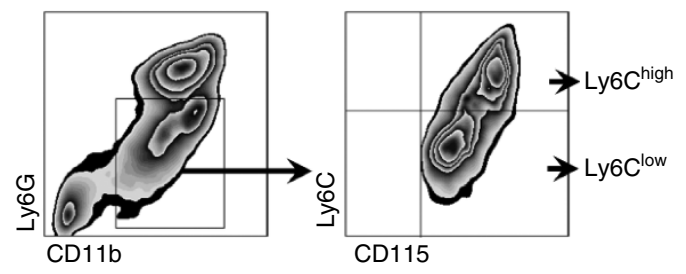

b
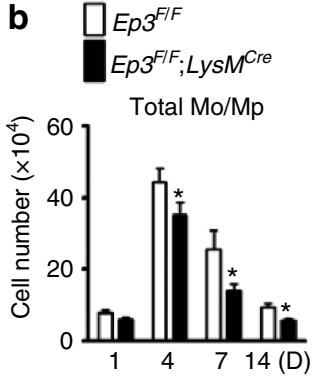

$\mathbf{f}$
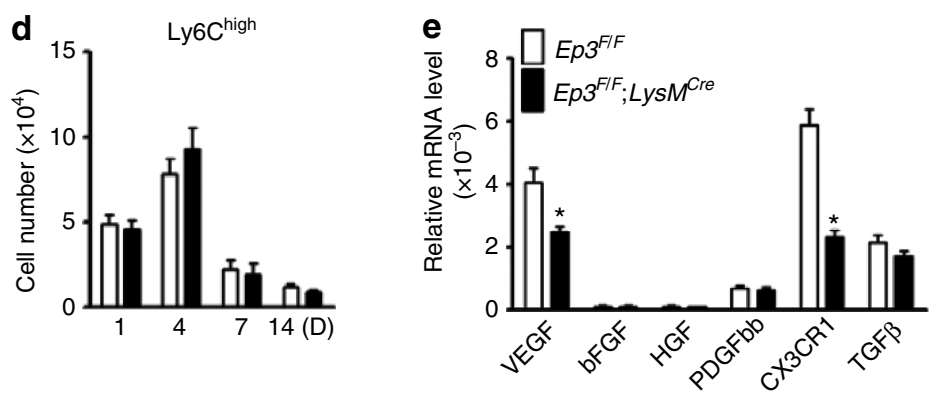

C
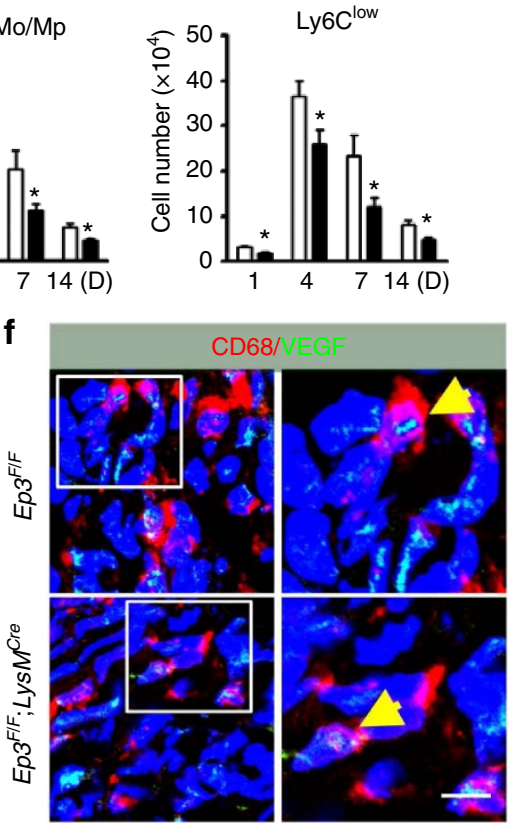

h
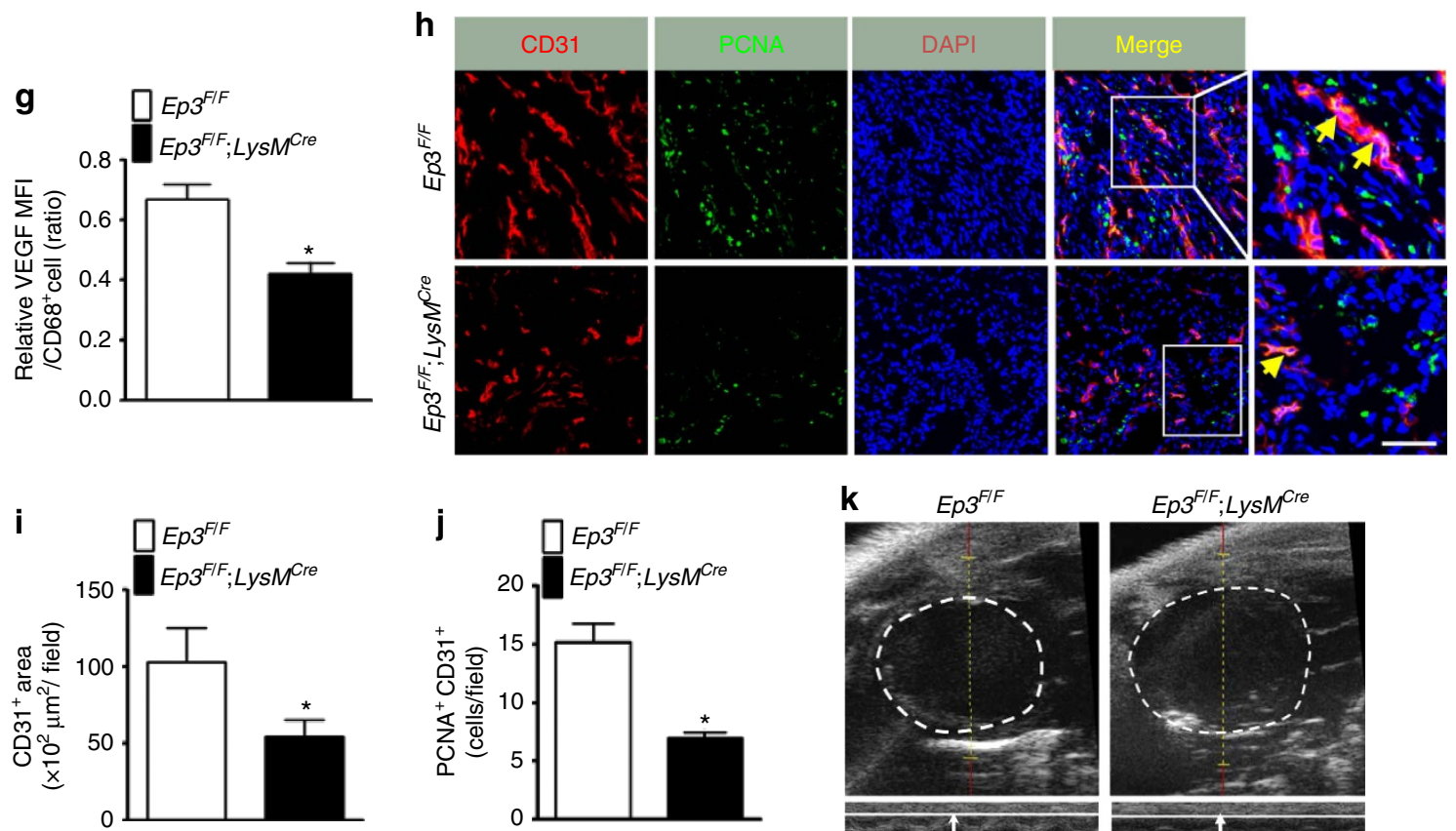

$E p 3^{F / F} ;$ LysM $M^{C r e}$
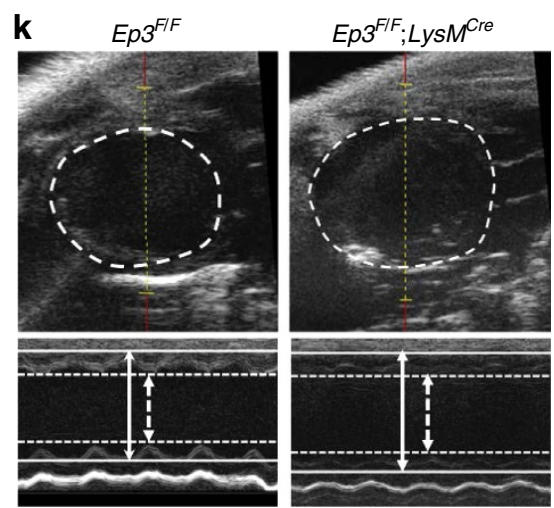
deficient Mps both in vivo and in vitro (Fig. 5a-c, Supplementary Fig. 15a-c). Additionally, Ep3 deletion suppressed mouse Mp migration in response to $\mathrm{PGE}_{2}$ (Fig. 5d). In human blood $\mathrm{CD} 14^{\mathrm{dim}} \mathrm{CD} 16^{+}$Mos are similar to reparative Ly6C $\mathrm{C}^{\text {low }}$ mouse Mos (ref. 20). Ep3 agonist sulprostone promoted the migration of human CD14 ${ }^{\text {dim }}$ CD16 ${ }^{+}$Mos, while Ep3 inhibitor L798106 suppressed $\mathrm{CD} 14^{\mathrm{dim}} \mathrm{CD} 16^{+}$Mo migration (Supplementary Fig. 16a,b). Likewise, Ep3 receptor was also involved in regulation of CX3CR1 expression in human Mos (Supplementary Fig. 16c). To investigate whether TGF $\beta 1$ signalling is involved in Ep3-mediated regulation of CX3CR1 and VEGF expression in Mos/Mps, we created an Mp-specific Ep3 $\alpha$ transgenic (Mp-EP3Tg) mouse model using the CD68 promoter (Supplementary Fig. 17a-d). Indeed, overexpression of Mp-Ep3 $\alpha$ induced activation of TGF $\beta 1$ signalling and elevated expression of both VEGF and CX3CR1 in Mps (Fig. 5e-h, Supplementary Fig. 18), and therefore increased $\mathrm{PGE}_{2}$-induced $\mathrm{Mp}$ migration (Fig. 5i), promoted infiltration of Ly6Clow Mos/Mps (Fig. 6a-c) and angiogenesis (Fig. 6d-f) in the infarcts, and facilitated cardiac recovery after MI (Fig. $6 \mathrm{~g}$ ). Interestingly, inhibition of TGF $\beta 1$ signalling markedly diminished the induction of VEGF and CX3CR1 expression (Fig. 5e-h) and augmented migration capacity in vitro in Ep3 $\alpha$-overexpressed Mps (Fig. 5i), retarded the increased Ly6Clow Mos/Mps accumulation in infarcts (Fig. 6c), and enhanced neovascularization in peri-infarct zones and cardiac function in $M p-E p 3 \alpha T g$ mice (Fig. 6d-g, Supplementary Table 5). We then tested whether the TGF $\beta 1$ pathway is implicated in $M p$-Ep3-mediated angiogenesis using an Mp/HUVEC co-culture system. As shown in Supplementary Fig. 19a-c, forced expression of Ep3 $\alpha$ in Mps increased VEGF expression at both the mRNA and protein levels, while the TGF $\beta 1$ signalling blocker SB525334 attenuated the elevated $V E G F$ expression in $\mathrm{Mp}$-Ep3Tg mice. Moreover, more sprouts and tubal structures from HUVECs containing beads were formed in culture with Mps from $M p$-Ep3Tg mice than with those from WT controls (Supplementary Fig. 19d-g). Again, these differences were lost by the addition of SB525334 (Supplementary Fig. 19d-g). Thus, Ep3-mediated reparative Ly6C ${ }^{\text {low }} \mathrm{Mo} / \mathrm{Mp}$ recruitment and neovascularization in infarcted hearts is dependent on TGF $\beta 1$ signalling.

CX3CR1 and VEGF are downstream targets of TGFß1. Through the PWMScan tool, 4 and 2 potential Smad binding elements (SBEs) were predicted in the promoter of murine $\mathrm{C} \times 3 \mathrm{cr} 1$ $(\mathrm{S} 1-4)$ and $\operatorname{Veg} f\left(\mathrm{S1}^{\prime}-2^{\prime}\right)$ genes, respectively (Fig. 7a,b). However, the fragments containing S3 and S4 SBEs in the promoter of Cx3crl were detected in the anti-Smad2/3 immunocomplex by Chip assay in mouse RAW264.7 cell line (Fig. 7c). Activation of
TGF $\beta 1$ signalling by co-transfection of the TGF $\beta$ receptor Alk 5 or addition of exogenous TGF $\beta 1$ markedly enhanced luciferase activities in the $C x 3 \mathrm{cr} 1$ promoter $(\mathrm{S} 3+\mathrm{S} 4)$ containing reportertransfected RAW264.7 cells, both of which could be specifically blocked by a TGF $\beta 1$ inhibitor (Fig. 7d). Mutation of either S3 or S4 significantly weakened the TGF $\beta 1$ and Alk5 co-transfectioninduced luciferase activities in RAW264.7 cells as compared to these of WT fragment (S3+S4) transfected cells (Fig. 7e). Moreover, Ep3 inhibition also suppressed S3 + S4 promoter-driven activity in a dose-dependent manner (Fig. 7f). Similarly, two SBEs $\left(\mathrm{S}^{\prime}{ }^{\prime}\right.$ and $\left.\mathrm{S} 2^{\prime}\right)$ were identified in the VEGF promoter region by Chip and luciferase assays (Fig. $7 \mathrm{~g}-\mathrm{i}$ ) in RAW264.7 cells. Moreover, 1 and 5 potential SBEs were predicted in the promoter of human CX3CR1 (hS) and VEGF genes (hS1'-hS5', Fig. $7 \mathrm{j}, \mathrm{k})$, respectively. Using a human THP-1 cell line, we identified one (hS, Fig. 7l-n) and three (hS3' ${ }^{\prime}$ hS5 $5^{\prime}$, Fig. 7o-q) functional SBEs in promoter of human CX3CR1 and VEGF genes, respectively. Therefore, activation of the EP3 receptor induces CX3CR1 and VEGF expression in Mps through TGF $\beta 1$ signalling and subsequently promotes Ly $6 \mathrm{C}^{\mathrm{low}}$ accumulation after $\mathrm{MI}$ and peri-infarct angiogenesis (Fig. 7r).

\section{Discussion}

$\mathrm{PGE}_{2}$ is implicated in the regulation of multiple aspects of inflammation by tuning the functions of different immune cells, including $\mathrm{Mos} / \mathrm{Mps}^{21}$. Mos/Mps are a dominant source of $\mathrm{PGE}_{2}$ at sites of inflammation ${ }^{22}$. In mice, Mos/Mps can be divided into two subsets based on the expression level of Ly6C (pro-inflammatory Ly6 $\mathrm{C}^{\text {high }}$ and less inflammatory and patrolling Ly6C $\left.{ }^{\text {low }}\right)^{6}$. In humans, $\mathrm{CD} 14^{+} \mathrm{CD} 16^{-}$Mos are similar to murine Ly6Chigh cells, while CD14 ${ }^{\text {dim }}$ Mos are similar to murine Ly6C ${ }^{\text {low }} \mathrm{Mos}^{20}$. We found that Ep3 mediates recruitment and reparative function of $\mathrm{Ly} 6 \mathrm{C}^{\text {low }} \mathrm{Mo} / \mathrm{Mp}$ in inflamed tissues. Activation of the Ep3 receptor upregulates expression of the $\mathrm{Mp}$ chemokine receptor CX3CR1, which mediates Ly6Clow Mo infiltration in hearts after $\mathrm{MI}^{6}$. In addition, the Mo/Mp Ep3 receptor also mediates expression of the pro-angiogenic factor VEGF, which stimulates wound healing after MI by enhancing neovascularization in peri-infarct zones in mice. In agreement with our observations, tumour-derived $\mathrm{PGE}_{2}$ promotes differentiation of tumour-associated suppressive Mps (M2-like $\mathrm{Mps}$ ) from the Mos infiltrated in tumours ${ }^{23}$. And the $\mathrm{PGE}_{2}-E p 3$ axis also mediates recruitment and maturation of mast cells upon repeated allergen challenges ${ }^{24}$.

The Ep3 receptor mediates multiple cellular cascades by activating different types of heterotrimeric $G$ proteins, including $\mathrm{G} \alpha \mathrm{s}, \mathrm{G} \alpha \mathrm{i}$ and $\mathrm{G} \alpha 12 / 13$ (refs 25,26). We recently discovered that activation of the Ep3 receptor augments Rho/ROCK-dependent

Figure 3 | Ep3 deletion retards cardiac recovery after MI in mice. (a) Gating strategy for CD11b ${ }^{+}$CD115 ${ }^{+}$Ly6G ${ }^{-}{\text {Ly } 6 C^{\text {high }}}$ and $C D 11 b^{+}$CD115 ${ }^{+}$Ly6G ${ }^{-}$ Ly6Clow Mos/Mps in hearts after left anterior descending (LAD) artery ligation in mice. (b-d) Effect of Ep3 deletion on recruitment of total Mos/Mps (b), Ly6Clow $(\mathbf{c})$ and Ly6Chigh $(\mathbf{d})$ Mos/Mps in injured hearts of mice after MI. Data represent mean \pm s.e.m. ${ }^{\star} P<0.05$ versus Ep $3^{F / F}$ (unpaired two-tailed $t$ test); $n=5$-8. (e) mRNA expression levels of VEGF, fibrolast growth factor (FGF), hepatocyte growth factor (HGF), platelet-derived growth factor-bb $(P D G F b b)$, and CX3CR1 in Mos/Mps sorted from hearts in $E p 3^{F / F}$ and $E p 3^{F / F} ;$ Lys $M^{C r e}$ mice at day 14 post MI. Data represent mean \pm s.e.m. ${ }^{\star} P<0.05$ versus $E p 3^{F / F}$ (unpaired two-tailed $t$-test); $n=10$. (f) Representative immunostaining for CD68 (red) and VEGF (green) in peri-infarct zones of hearts from Ep $3^{F /}$ $F_{\text {and }} E p 3^{\mathrm{F} / \mathrm{F}} ; \mathrm{LyS} \mathrm{M}^{\mathrm{Cre}}$ mice at day 14 post MI. The solid box outlines the region enlarged to the right. Yellow arrow, $\mathrm{CD} 68^{+} / \mathrm{NEGF}{ }^{+}$cell. Scale bar, $5 \mu \mathrm{m}$. (g) Quantitation of VEGF signalling in $\mathrm{CD} 8^{+}$cells in injured hearts as shown in f. Data represent mean \pm s.e.m. ${ }^{\star} P<0.05$ versus Ep3 ${ }^{F / F}$ (unpaired twotailed $t$-test); $n=5$. (h) Representative immunostaining of CD31 (red) and proliferating cell nuclear antigen (PCNA, green) in peri-infarct zones of hearts from $E p 3^{F / F}$ and $E p 3^{F / F} ;$ Lys $M^{C r e}$ mice at day 14 post MI. The solid box outlines the region enlarged to the right; yellow arrow, CD31 ${ }^{+} / \mathrm{PCNA}+$ cell. Scale bar, $20 \mu \mathrm{m}$. (i,j) Quantitation of $\mathrm{CD}_{1} 1^{+}$areas $(\mathbf{i})$ and PCNA ${ }^{+} \mathrm{CD} 31^{+}$cells $(\mathbf{j})$ in injured hearts as shown in $\mathbf{h}$. Data represent mean \pm s.e.m. ${ }^{\star} P<0.05$ versus $E p 3^{F / F}$ (unpaired two-tailed $t$-test); $n=7$. (k) Representative echocardiography images with $M$-mode views of infarcted hearts from $E p 3^{F / F} ; L^{2}{ }^{2} M^{C r e}$ and $\mathrm{Ep}^{\mathrm{F} / \mathrm{F}}$ mice on day 14 after MI. Arrows and lines mark left ventricular inner diameters (LVID) in systole (dashed) and diastole (firm). (I) Cardiac function of $E p 3^{F / F} ; L y s M^{C r e}$ and $E p 3^{F / F}$ mice at different timepoints after MI. EF, ejection fraction. ${ }^{\star} P<0.05$ versus Ep $3^{F / F}$ (unpaired two-tailed $t$-test); $n=9-13$. 

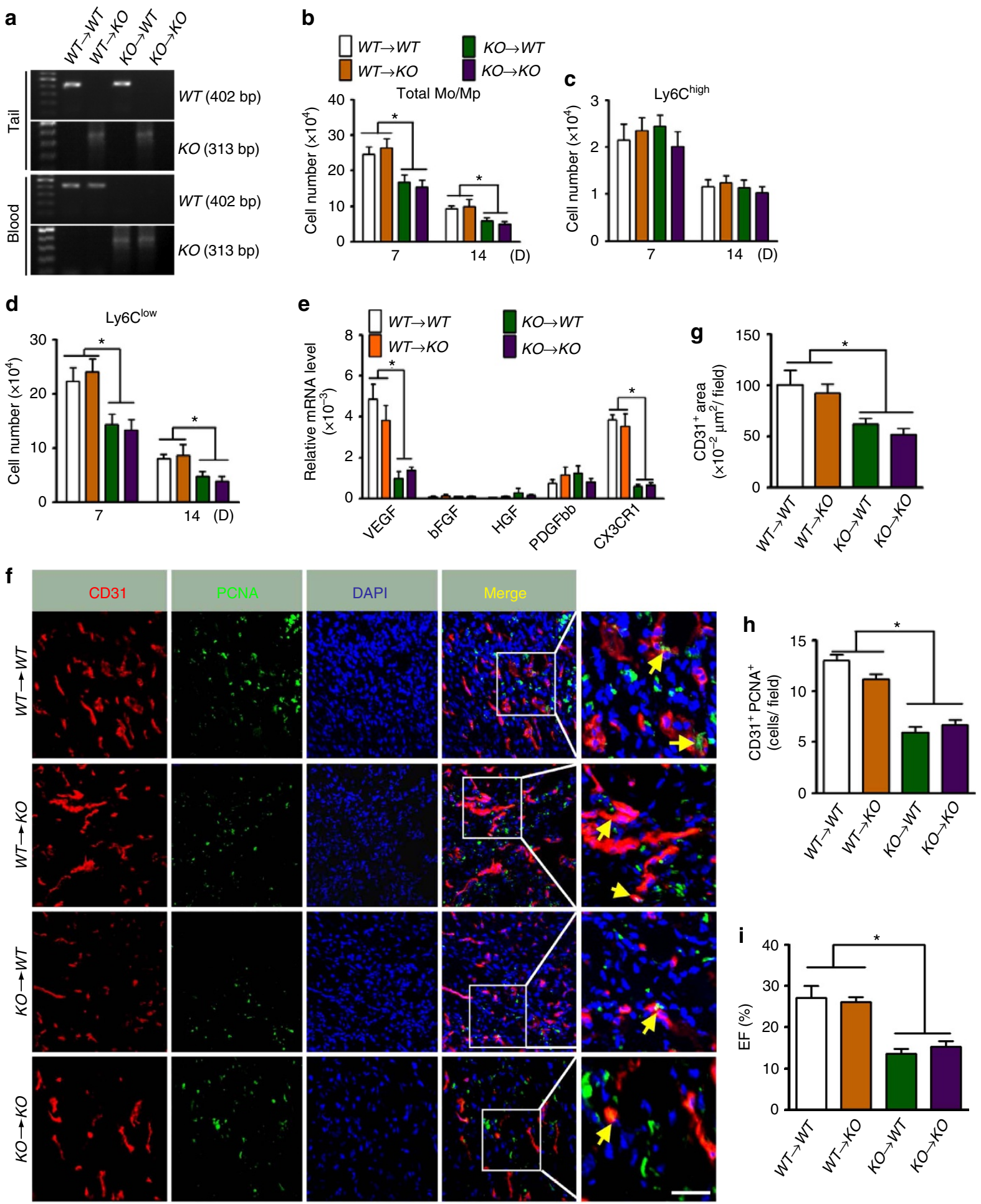

Figure 4 | BMT ameliorates impaired cardiac function after MI in EP3 KO mice. (a) Bone marrow transplantation (BMT) between Ep3 KO and WT mice was confirmed by genotyping. (b-d) Recruitment of total Mos/Mps (b), Ly6Chigh (c) and Ly6Clow (d) Mos/Mps in injured hearts in chimeric mice that underwent BMT. Data represent mean \pm s.e.m. ${ }^{\star} P<0.05$ as indicated (unpaired two-tailed $t$-test); $n=6-7$. (e) mRNA expression levels of VEGF, FGF, HGF, $P D G F b b$ and $C X 3 C R 1$ in Mos/Mps sorted from hearts from BMT chimeric mice at day 14 post MI. Data represent mean \pm s.e.m. ${ }^{\star} P<0.05$ as indicated (unpaired two-tailed $t$-test) $; n=4$. (f) Representative immunostaining of CD31 (red) and PCNA (green) in peri-infarct zones of hearts from BMT chimeric mice at day 14 post MI. The solid box outlines the region enlarged to the right. Yellow arrow, CD31+ $/$ PCNA ${ }^{+}$cells. Scale bar, $20 \mu \mathrm{m}$. ( $(\mathbf{g}, \mathbf{h}) \mathrm{Quantitation}$ of $\mathrm{CD}_{1}{ }^{+}$areas $(\mathbf{g})$ and $\mathrm{PCNA}{ }^{+} \mathrm{CD} 31^{+}$cells $(\mathbf{h})$ in injured hearts as shown in $\mathbf{f}$. Data represent mean \pm s.e.m. ${ }^{\star} P<0.05$ as indicated (unpaired two-tailed $t$-test); $n=6-7$. (i) Cardiac function of BMT chimeric mice at day 14 after MI. EF, ejection fraction. Data represent mean \pm s.e.m. ${ }^{\star} P<0.05$ as indicated (unpaired two-tailed $t$-test); $n=8-11$. 


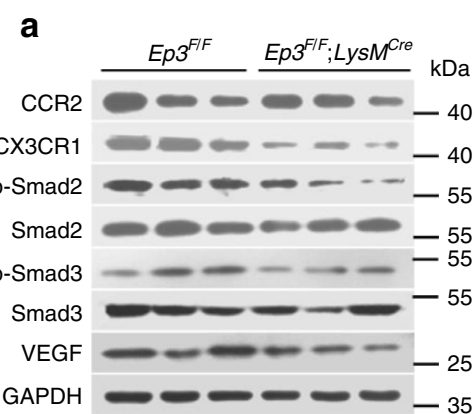

\section{b}

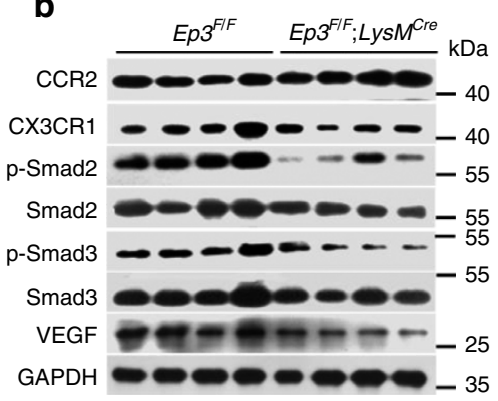

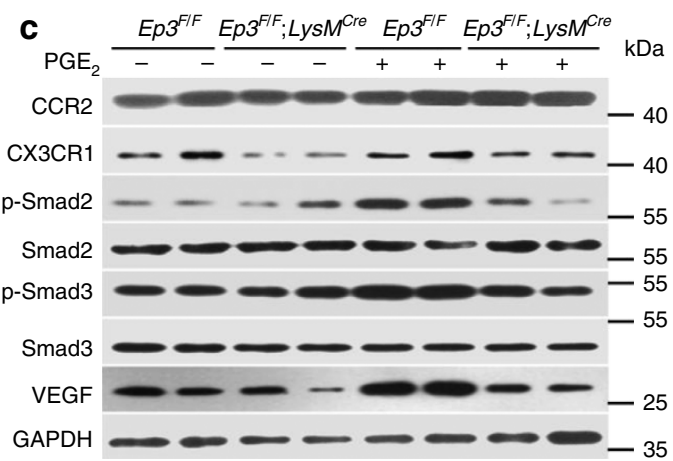

d
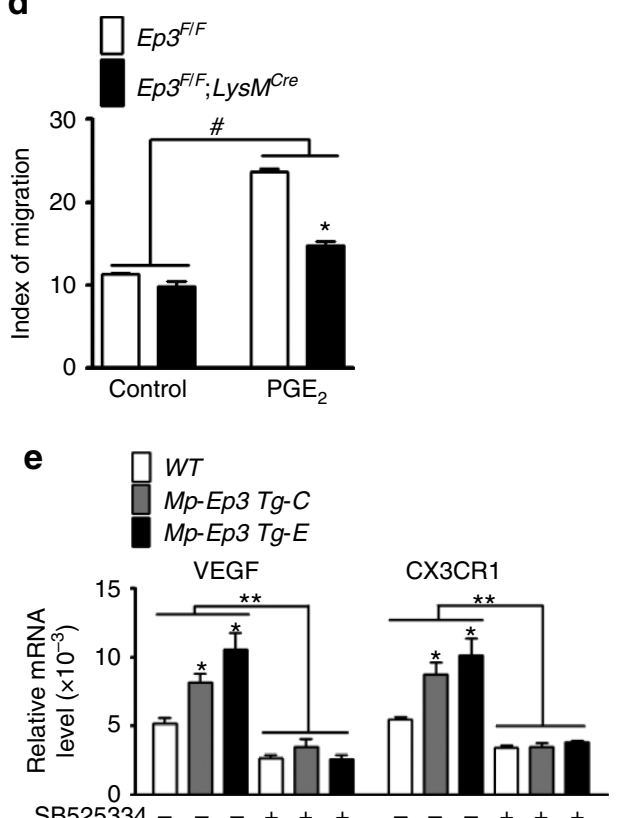

f

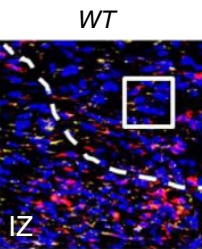

$M p-E p 3 T g-C$

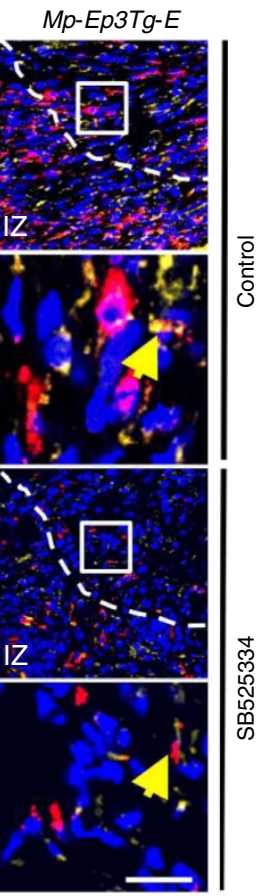

g

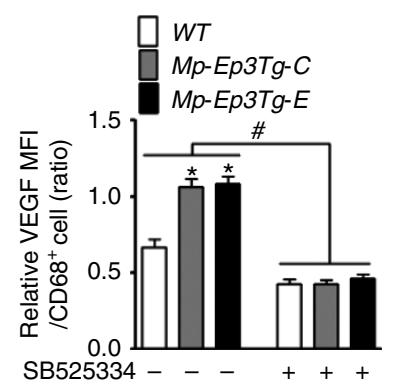

h

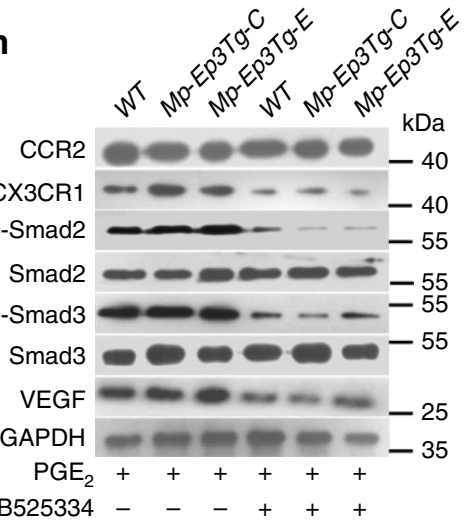

i

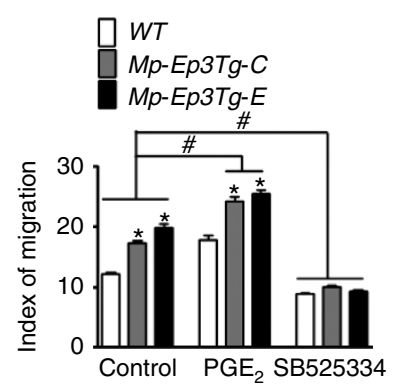

Figure 5 | Ep3 regulates CX3CR1 and VEGF expression through TGFß1 signalling. (a,b)Western blot analysis of CCR2, CX3CR1, phospho-Smad2, phospho-Smad3 and VEGF in Mos/Mps from Ep $3^{\mathrm{F} / \mathrm{F}}$ and $E p 3^{\mathrm{F} / \mathrm{F}} ; \mathrm{Lys} \mathrm{M}^{\mathrm{Cre}}$ mice at $16 \mathrm{~h}$ (a) and $96 \mathrm{~h}$ (b)after a zymosan challenge. (c) Western blot analysis of CCR2, CX3CR1, phospho-Smad2, phospho-Smad3 and VEGF in cultured Mps with or without PGE 2 stimulation. (d) Effect of Ep3 deletion on Mp migration in response to $P G E_{2}$. Data represent mean \pm s.e.m. ${ }^{\star} P<0.05$ versus $E p 3^{F / F},{ }^{\#} P<0.05$ as indicated (unpaired two-tailed $t$-test); $n=5$. (e) Effect of TGF $\beta 1$ blocker SB525334 on Ep3-mediated VEGF and CX3CR1 mRNA expression in cultured Mps. Mp-Ep3Tg, Mp-specific Ep3 $\alpha$ transgenic mice. Data represent mean \pm s.e.m. ${ }^{\star} P<0.05$ versus wild-type (WT), ${ }^{\star \star} P<0.01$ versus indicated (unpaired two-tailed $t$-test); $n=5$. (f) Representative immunostaining of $C D 68$ (red) and VEGF (green) in peri-infarct zones of hearts from Mp-Ep3Tg mice at day 14 post MI. The solid box outlines the region enlarged below. Yellow arrow, $\mathrm{CD} 68^{+} / \mathrm{VEGF}^{+}$cells. Scale bar $20 \mu \mathrm{m}$. IZ, infarct zone. (g) Quantitation of VEGF signalling in $\mathrm{CD}^{+} 8^{+}$cells in injured hearts as shown in $\mathbf{f}$. Data represent mean \pm s.e.m. ${ }^{\star} P<0.05$ versus WT, ${ }^{\#} P<0.05$ as indicated (unpaired two-tailed $t$-test); $n=5$. (h) Effect of SB525334 on Ep3-mediated VEGF and CX3CR1 protein expression in cultured Mps. (i) Effect of SB525334 on $\mathrm{PGE}_{2} /$ Ep3-mediated Mp migration in vitro. Data represent mean \pm s.e.m. ${ }^{\star} P<0.05$ versus $W T$, ${ }^{P} P<0.05$ as indicated (unpaired two-tailed $t$-test); $n=4-6$. 


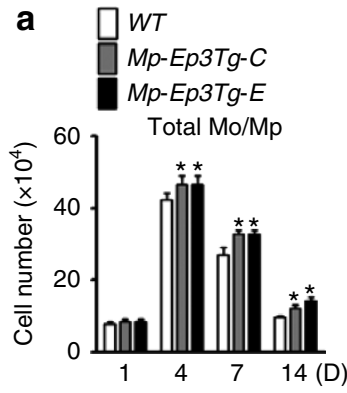

b

d
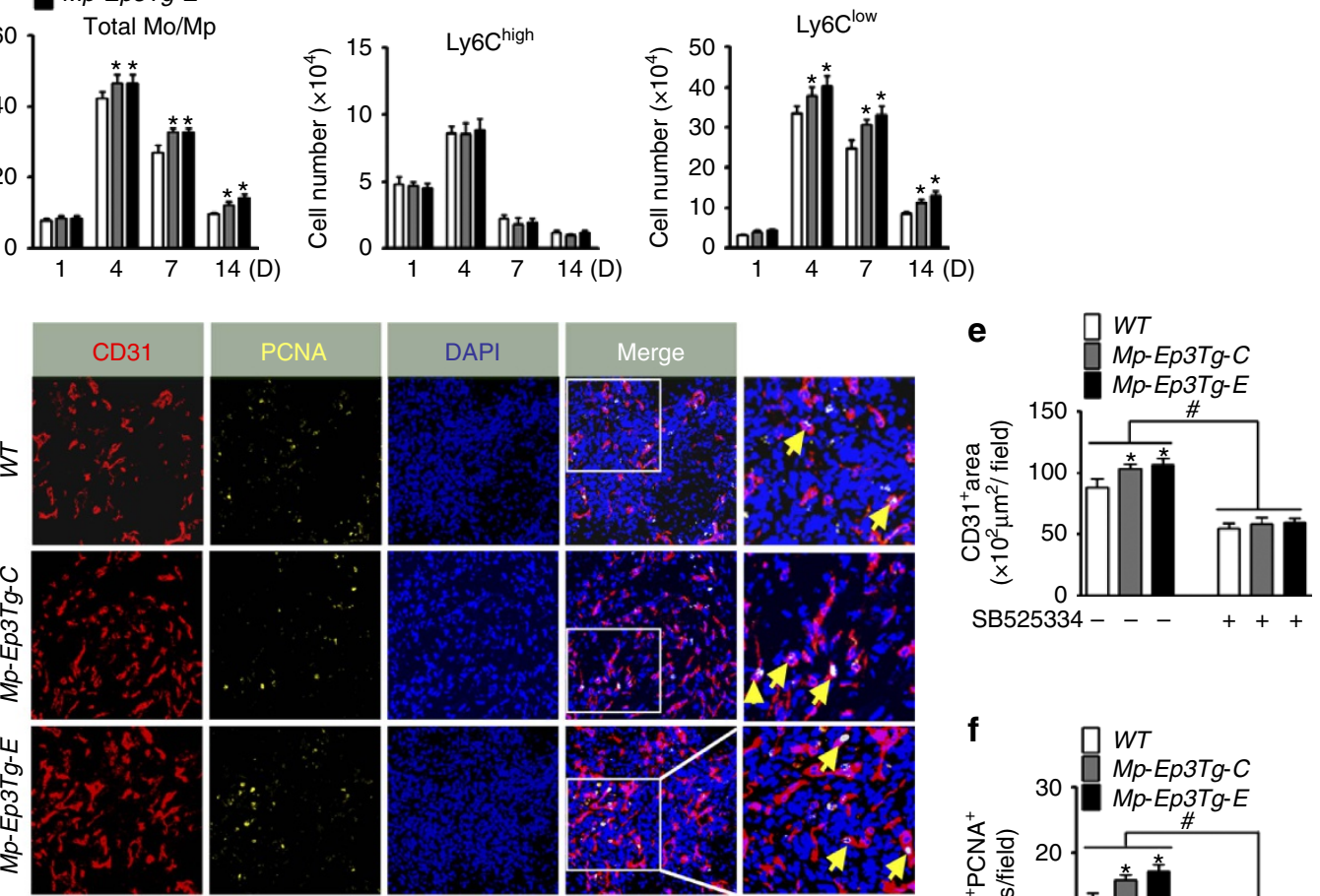

SB525334
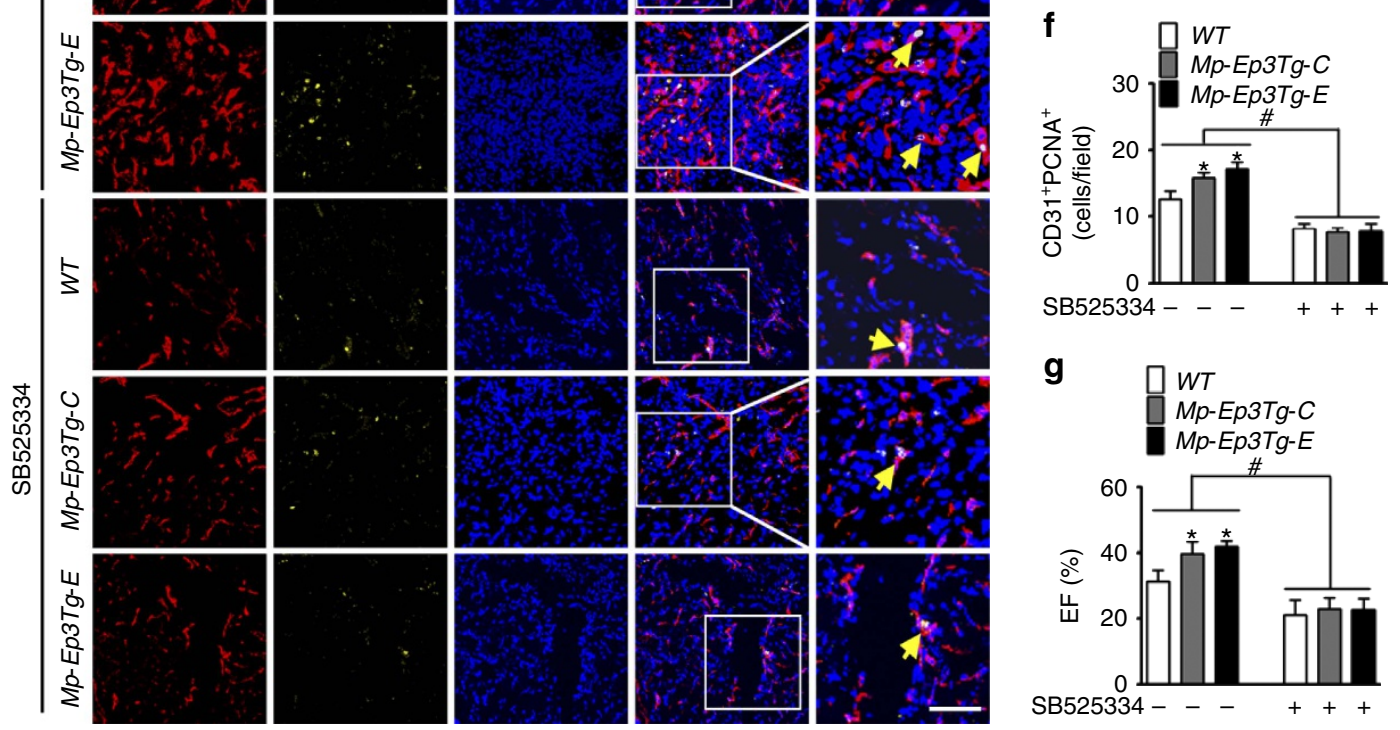

Figure 6 | Ep3 overexpression in Mos/Mps improves cardiac recovery after MI in mice. (a-c) Effect of Ep3 overexpression on recruitment of total Mos/ Mps (c), Ly6Chigh, (d) and Ly6C low Mos/Mps in injured hearts mice after MI. Data represent mean \pm s.e.m. ${ }^{\star} P<0.05$ versus WT (unpaired two-tailed $t$-test); $n=5-7$. (d) Representative immunostainings of CD31 (red) and PCNA (yellow) in peri-infarct zones of hearts from WT, Mp-Ep3Tg-C and Mp-Ep3Tg$E$ mice at day 14 post-MI. The solid box outlines the region enlarged to the right; yellow arrow, CD31+/PCNA ${ }^{+}$cell. Scale bar, $20 \mu \mathrm{m}$. (e,f) Quantitation of $\mathrm{CD}_{1}{ }^{+}$areas $(\mathbf{e})$ and $\mathrm{PCNA}{ }^{+} \mathrm{CD} 31^{+}$cells $(\mathbf{f})$ in injured hearts, as shown in $\mathbf{d}$. Data represent mean \pm s.e.m. ${ }^{\star} P<0.05$ versus $\mathrm{WT},{ }^{\#} P<0.05$ as indicated(unpaired two-tailed $t$-test); $n=6$. (g) Cardiac function of WT, Mp-Ep3Tg-C and Mp-Ep3Tg-E mice at day 14 after MI. Data represent mean \pm s.e.m. ${ }^{\star} P<0.05$ versus $W T,{ }^{\#} P<0.05$ as indicated (unpaired two-tailed $t$-test); $n=7-8$.

TGF $\beta 1 /$ Smad2/3 signalling to facilitate hypoxia-induced vascular fibrosis by coupling with $\mathrm{G} \alpha 12$ in pulmonary arterial smooth muscle cells ${ }^{19}$. Indeed, Ep3 deficiency also results in decreased Rho activity and subsequent suppression of TGF $\beta 1$ signalling in both peritoneal and infarcted myocardium-derived Mps, whereas forced expression of the Ep3 receptor in Mos/Mps amplified TGF $\beta 1 / \mathrm{Smad} 2 / 3$ signalling. Furthermore, we identified conservative SBEs in the promoter regions of both human and mouse CX3CR1 genes coding a chemokine receptor for CX3CL1, which governs Ly6 ${ }^{\text {low }}$ Mo migration into inflammation sites ${ }^{6}$. Thus, the Ep3 receptor regulates Ly6 $\mathrm{C}^{\text {low }} \mathrm{Mo} / \mathrm{Mp}$ recruitment into the ischaemic myocardium through the TGF $\beta 1 / C X 3 C R 1$ pathway. Interestingly, CX3CR1/CX3CL1 interactions confer a vital survival signal for Mos/Mps, whose complete disruption causes death of peripheral $\mathrm{Mos}^{27}$ and renal resident $\mathrm{Mps}^{28,29}$ and forms cells in atherosclerosis plaques ${ }^{27}$. We failed to observe marked death and/or apoptosis of peripheral Mos or Mps in the injured myocardium in $M p$-Ep3-deficient mice with reduced expression of CX3CR1 in Mos/Mps, which is consistent with the phenotypes presented in CX3CR1 heterozygous mice $\left(C X 3 C R 1^{G F P} /+\right)^{27,30}$. In addition, CX3CR1 deficiency appears to impair reparative functions of macrophages (pro-fibrosis, proangiogenesis, and phagocytosis) in a variety of disease processes, such as skin wound healing ${ }^{31-33}$. In an experimental MI mouse model, Mo/Mp Ep3 deletion retarded myocardial healing by inhibiting peri-infarct angiogenesis. Further mechanistic analysis revealed that Ep3 receptor activation in Mos/Mps promotes TGF $\beta 1 /$ Smad pathway-derived VEGF 
a

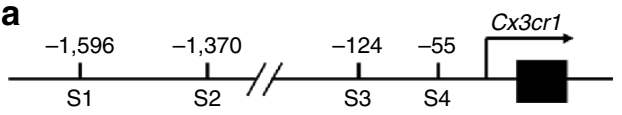

C

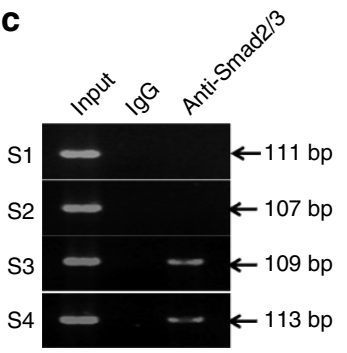

f

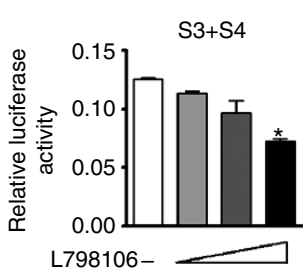

j

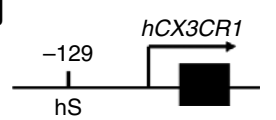

I

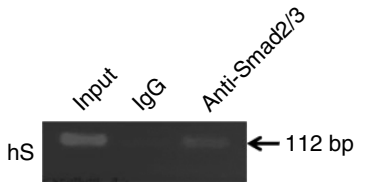

p

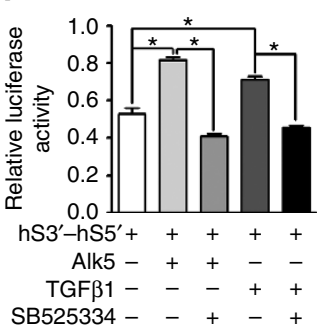

d

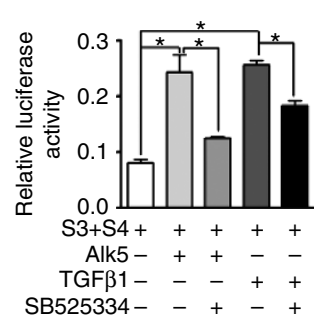

g

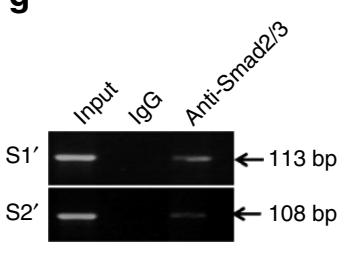

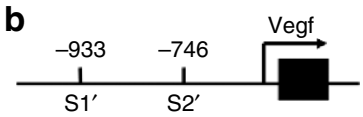

e

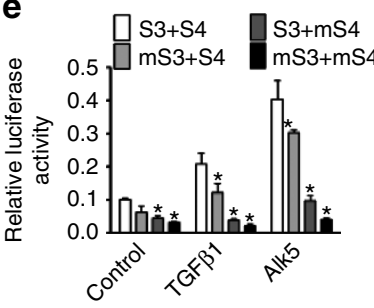

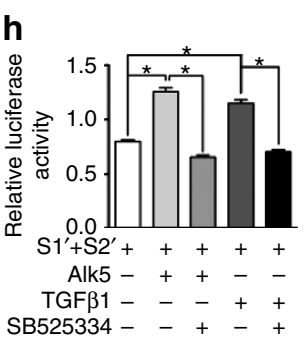

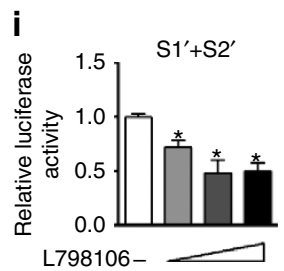

L798106 - k

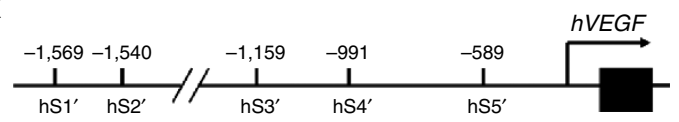

m
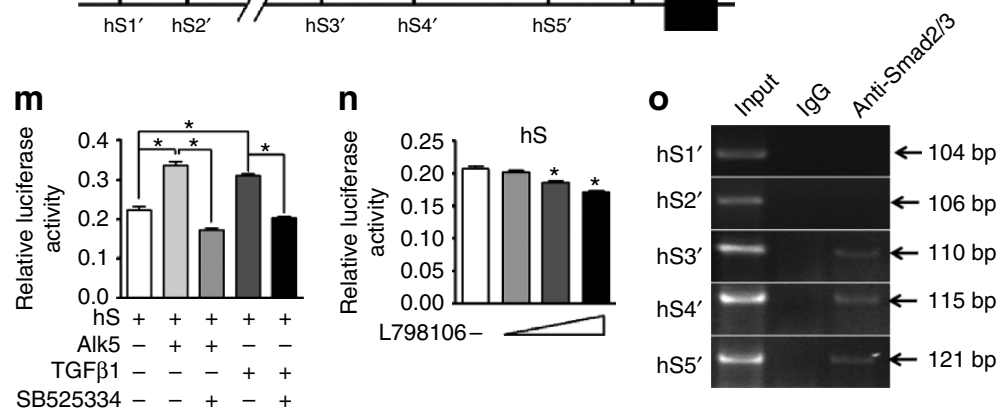

q

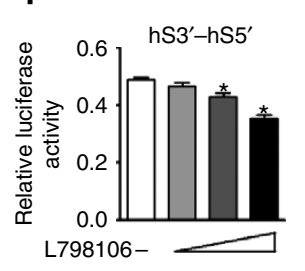

$\mathbf{r}$

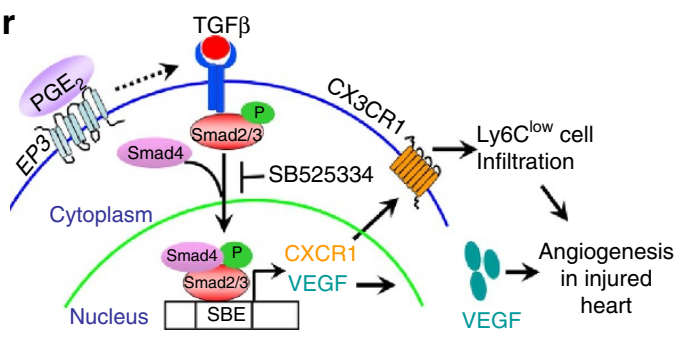

Figure 7 | CX3CR1 and VEGF are TGFß1-targeting genes in mice and humans. (a,b) Schematic illustration of predicted Smad binding elements (SBEs) in the promoter of murine $\mathrm{Cx} 3 \mathrm{crl}$ (a) and Vegf (b) genes. S = SBE. (c) Gel electrophoresis of PCR-amplified SBE-containing fragments in the promoter of the murine $\mathrm{Cx3}$ cr1 gene using anti-Smad2/3 immunoprecipitation. (d) Effect of the TGF $\beta 1$ blocker SB525334 on S3 + S4 fragment-mediated luciferase activity. ${ }^{\star} P<0.05$ as indicated (unpaired two-tailed $t$-test); $n=4$. (e) Effect of mutations of S3 (mS3) and S4 (mS4) on S3 + S4 fragment-mediated transcription activity. ${ }^{\star} P<0.05$ versus the S3 + S4 group, $n=4$. (f) Effect of L-798,106 on S3+S4 fragment-mediated luciferase activity in RAW264.7 cells. ${ }^{\star} P<0.05$ versus no treatment (unpaired two-tailed $t$-test); $n=4$. $(\mathbf{g})$ Gel electrophoresis of PCR-amplified SBE-containing fragments in the promoter of the murine Vegf gene using anti-Smad2/3 immunoprecipitation. (h) Effect of SB525334 on the functional SBEs $\left(\mathrm{S1}^{\prime}+\mathrm{S} 2^{\prime}\right)$ in murine Vegf promoter. ${ }^{\star} P<0.05$ as indicated (unpaired two-tailed t-test); $n=4$. (i) Effect of $\mathrm{L} 798106$ on $\mathrm{S} 1^{\prime}+\mathrm{S} 2^{\prime}$ fragment-mediated luciferase activity in RAW264.7 cells. ${ }^{*} P<0.05$ versus no treatment (unpaired two-tailed $t$-test); $n=5$. (j,k) Schematic illustration of the predicted SBE(s) on the human CX3CR1 gene (hS, (j)) and the human VEGF gene $\left(h^{\prime},(\mathbf{k})\right.$ ). (I) Gel electrophoresis of PCR-amplified SBE-containing fragments in the promoter of the human CX3CR1 gene. (m) Effect of SB525334 on hS-mediated transcription activity in THP-1 cells. ${ }^{\star} P<0.05$ as indicated (unpaired two-tailed $t$-test); $n=5$. (n) Effect of L-798,106 on hSmediated transcription activity in THP-1 cells. ${ }^{\star} P<0.05$ versus no treatment (unpaired two-tailed $t$-test); $n=5$. (o) Gel electrophoresis of PCR-amplified SBE-containing fragments in the promoter of the human VEGF gene. (p) Effect of SB525334 on hS3'-hS5' fragment-mediated transcription activity in THP-1 cells. ${ }^{\star} P<0.05$ as indicated, $n=5$. (q) Effect of L-798,106 on hS3'-hS5' fragment-mediated transcription activity in THP-1 cells. ${ }^{*} P<0.05$ versus no treatment (unpaired two-tailed $t$-test); $n=5$. (r) Schematic diagram of EP3-mediated regulation of CX3CR1 and VEGF genes in Mos/Mps through TGF $\beta 1$. 
transcription. As previously reported ${ }^{34}$, we validated multiple conservative SBEs in the VEGF gene promoter in both mouse and human $\mathrm{Mp}$ cell lines, but not in infiltrated cardiac Mo/Mps due to technical difficulties. Taken together, the data suggest that activation of the Mo/Mp Ep3 receptor facilitates wound healing after MI by increasing CX3CR1-mediated Ly6C low Mo recruitment and VEGF-induced angiogenesis, which are TGF $\beta 1 /$ Smad pathway-dependent.

Cardiac $\mathrm{PGE}_{2}$ generation increases during acute $\mathrm{MI}^{17}$. Despite the expression of multiple $\mathrm{PGE}_{2}$ receptor subtypes in hearts, selective stimulation of the Ep3 receptor displays cardioprotection against ischaemia/reperfusion injury in different mammalian species ${ }^{35-38}$. For instance, pharmacological activation of Ep3 receptor reduces myocardial infarct size in rodents by activation of protein kinase $\mathrm{C}$ (PKC) and the opening of ATP-sensitive potassium channels in cardiomyocytes ${ }^{36,37}$. We found that inactivation of Ep3 in Mos/Mps impairs myocardial repair after acute ischaemia; in contrast, forced activation of Ep3-mediated signalling in Mos/Mps accelerates healing in mice after MI by increasing recruitment of reparative Mos/Mps and secretion of proangiogenic VEGF. Thus, Ep3 mediates cardiac protection against ischaemia, at least in part through activation of reparative Mos/Mps. In addition, Ep3 is also involved in regulation of lipid metabolism. Global Ep3 deletion promotes to diet-induced obesity and exaggerates ectopic lipid deposition such as in skeletal muscle in mice ${ }^{39,40}$. Hepatic Ep3 deficiency exacerbated atherosclerosis in hyperlidemic mice through suppression of biliary cholesterol secretion in mice ${ }^{41}$. However, Ep3 mediates vasoconstriction ${ }^{42}$, and genetic deletion of Ep3 receptor increases bleeding tendency ${ }^{38}$ and reduces atherosclerosis-related thrombosis ${ }^{43}$, suggesting Ep3 involvement in platelet activation. Therefore, the potential efficacy of specific activation of Ep3 receptor in cardiac recovery after MI requires further investigations.

In summary, we showed that the $\mathrm{PGE}_{2}-E p 3$ axis in $\mathrm{Mo} / \mathrm{Mp}$ exerts a beneficial effect on myocardial recovery in response to acute ischaemia through TGF $\beta 1$-mediated activation of reparative Ly6C ${ }^{\text {low }}$ Mos/Mps. These observations suggest Ep3 receptor and its downstream pathway maybe a promising therapeutic target for acute MI.

\section{Methods}

Mice. All animal procedures were approved by the Institutional Animal Care and Use Committee of the Institute for Nutritional Sciences, Chinese Academy of Sciences, as well as the number of animals to be used were approved based on the expected effects size. Ep $3^{\mathrm{F} / \mathrm{F}}\left(E p 3^{F / F}\right)^{44}$ and $E p 3$ knockout (KO) mice ${ }^{19}$ were maintained on C57BL/6 background, $L y s M^{C r e}$ transgenic mice (C57BL/6, The Jackson Laboratory) were crossed $E p 3^{F / F}$ to obtain $E p 3^{F / F} ; L y s M^{C r e}$ mice.

Echocardiography. High-resolution echocardiography imaging system (Vevo 770, Visual Sonics) were used with M-mode analysis to assess mouse cardiac function. Both male and female mice (10-14 weeks old) were anaesthetized by isoflurane inhalation (1-2\%) and heart rate was maintained at 350-500 b.p.m. The mitral valve leaflet was visualized and cardiac function was evaluated in the parasternal long axis view as previously described ${ }^{45}$

Macrophage-specific Ep3 $\alpha$ transgenic mice. Macrophage (Mp)-specific Ep3 $\alpha$ transgenic mice were generated as described previously ${ }^{46}$. Briefly, Ep $3 \alpha$ cDNA was cloned into the CD68 promoter-contained vector, and the successful construct was injected into C57BL6 zygotes to obtain founder mice.

Mouse model of myocardial infarction. Left anterior descending (LAD) ligation was used in both male and female mice (8-12 weeks old) to induce MI. Briefly, mice were anesthetized with isoflurane (2\%) using an induction chamber, and the LAD coronary artery was completely ligated to induce left ventricular ischaemia ${ }^{47}$.

Myocardial necrotic injury was assessed by nitro blue tetrazolium (NBT) staining as previously reported ${ }^{48}$. Briefly, hearts were collected 12 or $24 \mathrm{~h}$ after LAD ligation and then cut into slices about $2 \mathrm{~mm}$ thick. Slices were incubated in
$0.5 \mathrm{mg} \mathrm{ml}^{-1} \mathrm{NBT}$ in phosphate buffer at $\mathrm{pH} 7.4$ and $37^{\circ} \mathrm{C}$ for $20 \mathrm{~min}$. The unstainedportion (necrotic) could be separated from the dark blue stained (non-necrotic) region. The percentage of necrotic area was determined by dividing the weight of the necrotic tissue by the total weight and multiplying by 100 .

As for infarction analysis, hearts were dissected at day 14 after infusion of $10 \%$ evans blue $(100 \mu \mathrm{l})$ and frozen at $-20^{\circ} \mathrm{C}$ for $30 \mathrm{~min}$, then cut into 2 - $\mathrm{mm}$ thick slices from apex to base. The slices were incubated in $1 \%$ triphenyltetrazolium chloride at $37^{\circ} \mathrm{C}$ for $30 \mathrm{~min}$ to identify non-infarcted and infarcted areas. The normal myocardium was then recognized by red staining by triphenyltetrazolium chloride, while infarcted tissue presented as milky white. Infarct area was calculated as the ratio of the infarct area to the total slice areas previously described ${ }^{49}$.

Enzyme-linked immunosorbent assay. Serum was collected from retro-orbital blood from mice 14 days after MI induction. Levels of the heart failure marker brain natriuretic protein in serum was measured by enzyme-linked immunosorbent assay, according to the manufacturer's instructions (R\&D Systems).

Immunofluorescence staining. For immunofluorescence, heart sections $(8 \mu \mathrm{m})$ were incubated with primary antibodies against CD68 (Serotec, 1:200), CD301 (Biolegend, 1:200), PCNA (CST, 1:800), CD31 (RD, 1:200), VEGF (Proteintech, 1:200), SMA (Sigma, 1:500), CollagenI(Proteintech,1:200), THBS1( Proteintech, 1:200) and Alexa Fluor 488/594/633-conjugated secondary antibodies (Invitrogen, Carlsbad, CA, 1:1,000) for $1 \mathrm{~h}$ at room temperature, respectively. The embedded hearts were washed and stained with DAPI (Millipore, MA). Images were captured under a Olympus (FV1000) laser-scanning confocal microscope from each heart section for further analysis. Positive staining area and relative mean fluorescent intensity were measured using Image-Pro Plus software 6.0 (Media Cybernetics, Rockville, MD, USA $)^{50}$

For immunocytochemistry, primary Mos/Mps grown on slides were stimulated with or without $\mathrm{PGE}_{2}$ and then fixed with $4 \%$ paraformaldehyde. The slides were blocked in TBST containing $1 \%$ bovine serum albumin after washing, incubated with primary antibodies against CD68 (Serotec, 1:200) or VEGF (Proteintech, 1:200) overnight at $4{ }^{\circ} \mathrm{C}$, stained with a secondary antibody for $2 \mathrm{~h}$ at room temperature, and photographed using fluorescent microscopy as described above. At least five random images were taken in region of each slide and positive signalling was quantified as previously described ${ }^{51}$.

Bone marrow transplantation. Same sex BMT was performed as previously described $^{52}$. In brief, mice (6-8 weeks old) were euthanized and bone marrow (BM) cells were collected from femurs and tibias. Recipient mice were lethally irradiated (one 5.0-Gy dose and another 4-Gy dose administered $1.5 \mathrm{~h}$ apart) from a 137Cs source (MDS Nordion, Ottawa, Ontario, Canada) and transplanted with $5 \times 10^{6}$ donor $\mathrm{BM}$ cells via tail vein injection to reconstitute the hematopoietic system. Eight weeks after transplantation, BMT chimeric mice were used for experiments.

Fluorescence activated cell sorting analysis. Cells collected from mice were stained with fluorochrome-conjugated antibodies, according to the manufacturer's protocols. FITC-conjugated antibodies to CD11b (M1/70,1:50), Brilliant Violet 421-conjugated antibodies to CD115 (AFS98,1:50), APC-conjugated antibody to Ly6C (HK1.4,1:50), APC-CY7-conjugated antibody to Ly6G $(1 \mathrm{~A} 8,1: 50)$ were purchased from Biolegend. Flow cytometry was performed using a BD FACS Aria flow cytometry system (BD Biosciences, San Jose, CA), and data were analysed with FlowJo software. Total viable leukocyte number was determined with the trypanblue exclusion method. Leukocyte subpopulation numbers were calculated as total leukocytes multiplied by per cent cells within the selected population gated by FlowJo software (Tree Star, Ashland, Ore) and all FACS gates for fluorochromelabelled monoclonal antibodies were defined using appropriate isotype controls.

Peritoneal inflammation. Peritonitis was induced by intraperitoneal injection of zymosan ( $2 \mathrm{mg} \mathrm{ml}^{-1}$; Sigma-Aldrich, St Louis, MO). Peritoneal cells at different time points after the zymosan challenge were isolated for FACS analysis.

Cell extract and culture. Primary Mos/Mps were prepared from the abdominal cavity and cultured in 10\% FBS 1640 medium. A single-cell suspension from infracted hearts was prepared as previously described ${ }^{53}$. Briefly, the hearts were dissected, carefully cut into small pieces with fine scissors, and enzymatically digested with $450 \mathrm{U} \mathrm{ml}^{-1}$ of a cocktail of type I collagenase, $125 \mathrm{U} \mathrm{ml}^{-1}$ type IX collagenase, and $60 \mathrm{U} \mathrm{ml}^{-1}$ DNase I, and $60 \mathrm{U} \mathrm{ml}^{-1}$ hyaluronidase I-S ( Sigma, St Louis, MO) for $1 \mathrm{~h}$ at $37^{\circ} \mathrm{C}$. After digestion, the tissues were passed through $70-\mu \mathrm{m}$ cell strainers, washed, and stained with antibody for FACS analysis.

Western blot. Cells were extracted in lysis buffer containing protease inhibitors. Protein concentration was determined by the BCA method using the Pierce BCA Protein Assay Kit (Pierce, Rockford, IL). Equal quantities of proteins from total cell lysates were separated by $10 \%$ SDS-PAGE gel and probed with antibodies against CX3CR1 (1:500, Abcam), CCR2 (1:500, Abcam), p-Smad2 (1:1,000, CST), p-Smad3 
(1:1,000, CST), T-Smad3 (1:1,000, CST), T-Smad2/3 (1:1,000, CST), VEGF (1:1,000, Proteinteh), and GAPDH (1:2,000, CST), and then conjugated with an HRP-labelled secondary antibody in blocking buffer for $1-2 \mathrm{~h}$ at room temperature. Proteins were detected using an ECL detection kit (Super Signal West Pico Chemiluminescent Substrate), and signalling was quantified by image J software and presented as normalized arbitrary units. The uncropped scans of all western blots are supplied in Supplementary Fig. 20.

Macrophage (Mp) migration assay. Primary Mps (1000,000 cells per well) were pretreated with $10 \mathrm{nM} \mathrm{PGE}_{2}$ in serum-free DMEM media for $24 \mathrm{~h}$ and then seeded on cell culture inserts (Millicell-PCF, Millipore) with porous polycarbonate filters $(8-\mu \mathrm{m}$ pore size $)$ in 24 -well plates. DMEM with CX3CL1 $\left(20 \mathrm{ng} \mathrm{ml}^{-1}\right)$ was placed in the lower chamber at $37^{\circ} \mathrm{C}$ and $5 \% \mathrm{CO}_{2}$. After $6 \mathrm{~h}$, cells that migrated to the bottom well of the transwell chamber were fixed in paraformaldehyde (Thermo Scientific) and stained with DAPI for quantitation. The chemotactic index is the ratio of migrated Mps to total Mps.

Quantitative RT-PCR analysis. RNA was isolated from tissues and cells using the Trizol reagent (Invitrogen, Carlsbad, CA), and cDNA was synthesized by reverse transcription kits (Takara, Dalian, China). All mRNA expression levels were normalized by comparing them to the housekeeping gene GAPDH. All RT-PCR primers are listed in Supplementary Table 6.

Prediction of transcription-factor binding sites. The fragment between $-2,000$ and $\pm 500 \mathrm{bp}$ of the transcription start sites of the CX3CR1 and VEGF gene from the UCSC genome browser database was analysed for putative Smad binding element (SBE) motifs in TRANSFAC version 10.2 using PWMSCAN ${ }^{54}$. A match score with a $P$ value $<5 \times 10^{-6}$ was considered to indicate a high-confidence binding site prediction.

Chromatin immunoprecipitation (Chip) assays. Chip assays were conducted with a Magna ChIPTM A/G chromatin immunoprecipitation kit (Millipore) according to the manufacturer's protocol. In brief, RAW264.7 and THP-1 cells were stimulated for $24 \mathrm{~h}$ by TGF $\beta 1$. Cells were then cross-linked with formaldehyde ( $1 \%$ final concentration), and the pellet was lysed and sonicated to shear the chromatin into 200-1,000-bp fragments. The lysates were diluted using a chromatin dilution buffer. The chromatin extract was incubated with $10 \mu \mathrm{g}$ of rat anti-Smad $2 / 3$ antibody (Abcam) or rat IgG (negative control) at $4{ }^{\circ} \mathrm{C}$ with rotation overnight, and the antibody-antigen-DNA complex was collected by protein G-agarose. The immunocomplexes were washed and the protein-DNA complexes were eluted, and then proteinase $\mathrm{K}$ was used to reverse the cross-linking of proteinDNA complexes to free up DNA. DNA was purified with the DNA purification kit (Promega), dissolved in elution buffer, and used for quantitative PCR analysis. The primers used for amplification of SBE-containing fragmentsof CX3CR1 and VEGF genes are listed in Supplementary Table 7.

Luciferase reporter assay. Mouse macrophage cell line RAW264.7(ATCC TIB-71) and Human monocyte cell line THP-1(ATCC TIB-202) were tested every 3 months for mycoplasma contamination by performing a PCR on the cell supernatant. They were seeded into 24 -well plates and grown to $70 \%$ confluence. Luciferase reporter or control empty vector plasmids were co-transfected with RPL-TK (internal plasmid) (20:1) into cells by using a lipofectamine 2000 transfection reagent (Invitrogen). Cells were cultured in a medium containing 1\% FBS and lysed $24 \mathrm{~h}$ after transfection. The Ep3 antagonist L798106 (Cayman, $0.1-10 \mu \mathrm{mol}$ ) or TGF $\beta 1$ (Selleck, $10 \mathrm{ng} \mathrm{ml}^{-1}$ ) was used to treat cells overnight $16 \mathrm{~h}$ after transfection. Luciferase activities were monitored with a dual luciferase reporter assay kit (Promega).

Plasmid construction. Mouse Cx3cr1 promoter DNA fragments ( -453 to $124 \mathrm{bp}$ ) and Vegf promoter DNA fragments (-1,216 to $399 \mathrm{bp}$ ) were amplified from mouse macrophage genomic DNA by PCR. The two promoter fragments were subcloned into pGL3 (Promega). The reporter with the insert of a fragment of -453 to 124 of $C X 3 C R 1$ gene was named S3 + S4. Mutations were introduced into the third (mS3) and fourth (mS4) SBEs of the CX3CR1 promoter by QuikChange site-directed mutagenesis (Stratagene, La Jolla, CA). Human CX3CR1 promoter DNA fragments ( -196 to $107 \mathrm{bp})$ and VEGF promoter DNA fragments $(-1,278$ to $134 \mathrm{bp}$ ) were amplified and subcloned into the PGL3 vector for the reporter assay. The PCR primers for promoter subcloningare listed in Supplementary Table 8.

In vitro HUVEC fibrin gel bead assay. Human vascular endothelial cells (HUVECs), from PromoCell (C-12200) were mixed with dextran-coated Cytodex 3 microcarriers (GE) at a concentration of 400 HUVECs per bead in $1.5 \mathrm{ml}$ of EGM2 medium (Clonetics, Walkersville, MD). Beads with cells were shaken gently at $37^{\circ} \mathrm{C}$ and $5 \% \mathrm{CO}_{2}$ every $20 \mathrm{~min}$ for $4 \mathrm{~h}$. After incubating, beads with cells were transferred to a T2 tissue culture flask (Corning) and left overnight in $5 \mathrm{ml}$ of EGM-2 at $37^{\circ} \mathrm{C}$ and $5 \% \mathrm{CO}_{2}$. After that, beads with cells were washed three times with $1 \mathrm{ml}$ of EGM-2 and re-suspended at a concentration of 200 cell-coated beads per $\mathrm{ml}$ with $2.5 \mathrm{mg} \mathrm{ml}^{-1}$ of fibrinogen (Sigma) and 0.15 units ml $\mathrm{ml}^{-1}$ of aprotinin (Sigma). Five hundred microlitre of fibrinogen/bead solution was added to 0.625 units of thrombin (Sigma) in one well of a 24-well tissue culture plate. The fibrinogen/bead solution was allowed to clot for $5 \mathrm{~min}$ at room temperature and then at $37^{\circ} \mathrm{C}$ and $5 \% \mathrm{CO}_{2}$ for $20 \mathrm{~min}$. One millilitre of EGM-2 (which contains $2 \%$ FBS) with or without 0.15 units $\mathrm{ml}^{-1}$ aprotinin was added to each well and equilibrated with the fibrin clot for $30 \mathrm{~min}$ at $37^{\circ} \mathrm{C}$ and $5 \% \mathrm{CO}_{2} ; 10^{6}$ macrophage cells were plated on top of the clot. Assays were terminated at day 7 for immunostaining and imaging 55 .

PG extraction and analysis. Hearts collected from mice 14 days after MI were homogenized, and $500 \mu \mathrm{l}$ supernatant were used for PG extraction. Heart prostanoid metabolites were extracted and quantitated using liquid chromatography/mass spectrometry/mass spectrometry (LC/MS/MS) analyses. In brief, following internal standards $(2 \mu \mathrm{l}), 40 \mu \mathrm{l}$ citric $(1 \mathrm{M})$ and $5 \mu \mathrm{l}$ BHT were added to the sample and then strenuously vibrated with $1 \mathrm{ml}$ solvent (normal hexane: ethyl acetate, 1:1) for $1 \mathrm{~min}$. After centrifugation $\left(6,000 \mathrm{~g} \mathrm{~min}^{-1}\right)$ for $10 \mathrm{~min}$, the supernatant organic phase was collected and dried under a gentle stream of nitrogen, dissolved in $100 \mu 110 \%$ acetonitrile in water. Production was normalized to total protein.

Isolation of human blood Mos. Human peripheral blood mononuclear cells (PBMCs) were purchased from Zenbio and resuspended $\left(10^{6} \mathrm{cells} / 10 \mu \mathrm{l}\right)$ in sorting buffer (2 mM EDTA, 0.5\% BSA in PBS) and stained with anti-human antibodies (Biolegend) specific for anti-CD14 and anti-CD16. Stained cells were filtered $(70 \mu \mathrm{m})$ and sorted on a BD FACSAria II cell sorter using appropriate colour compensation for correcting spectral overlap and autofluorescence. Isolated $\mathrm{CD} 14^{\mathrm{dim}} \mathrm{CD} 16^{+}$Mos were used for subsequent experiments.

Mo adoptive transfer. For zymosan-induced peritonitis mouse model, Mo subsets were sorted from spleens of $\mathrm{EP}^{\mathrm{F} / \mathrm{F}}$ and $\mathrm{EP} 3^{\mathrm{F} / \mathrm{L}} \mathrm{Lys} \mathrm{S}^{\mathrm{Cre}}$ mice, then labelled with cell proliferation dye eFluor 670 (eBioscience) and injected into C57/BL6 mice $6 \mathrm{~h}$ after zymosan challenge by tail vain.

For MI model, Mo subsets were collected from $\mathrm{CD} 45.2^{+}$mice as previously described $^{10}$, and injected into CD $45.1^{+}$mice on day 3 post MI. Infarcted hearts at day 6 post MI were dissected for preparation of single-cell suspension and flow cytometric analysis.

Histological analysis. The infacted hearts were collected at indicated time points and embedded in the paraffin. The sections $(6 \mu \mathrm{m})$ were stained with hematoxylineosin and Masson trichrome (Sigma). IPP software was used to analyse the necrotic area or collagen density as described ${ }^{56}$

Isolation of neutrophils and function assay. APC-conjugated antibody to CD11b (M1/70),PE-conjugated antibody to CD45(30-F11) and APC-CY7conjugated antibody to Ly6G (1A8)were purchased from Biolegend. Neutrophils were sorted from injured hearts by FACS and calculated by FlowJo software (Tree Star, Ashland, Ore). Ly6G (ab25377) were purchased for immunofluorescence in mice heart after MI. For reactive oxygen species (ROS) measurement, purified neutrophils were loaded for $20 \mathrm{~min}$ at $37^{\circ} \mathrm{C}$ with dichloro-dihydrofluorescein diacetate (DCFH-DA). Cells were washed and then production of ROS was quantified via flow cytometry by measurement of DCF. To detect intracellular myeloperoxidase, $5 \times 10^{4}$ neutrophils were lysed with TBS containing $0.2 \%$ Triton $\mathrm{X}-100(50 \mathrm{ml})$ and the myeloperoxidase activity was measured as previously described $^{57}$.

Statistics. Prism software (GraphPad Prism version 5.0) was used for statistical analysis. Results are shown as mean \pm s.e.m. Two-tailed student's $t$-testing and oneor two-way ANOVA with Bonferroni post-hoc analyses were used for comparisons between different groups. A $P$ value less than 0.05 was considered significant. Sample sizes were designed with adequate power according to the literature and our previous studies. Randomization and blinding strategy was used whenever possible.

Data availability. All the data supporting the findings of this study are either included in the manuscript and its Supplementary Information Files, or can be obtained from the corresponding author upon reasonable request.

\section{References}

1. Dutta, P. \& Nahrendorf, M. Monocytes in myocardial infarction. Arterioscler. Thromb., Vasc. Biol. 35, 1066-1070 (2015).

2. van Amerongen, M. J., Harmsen, M. C., van Rooijen, N., Petersen, A. H. \& van Luyn, M. J. Macrophage depletion impairs wound healing and increases lef ventricular remodeling after myocardial injury in mice. Am. J. Pathol. 170, 818-829 (2007). 
3. Frantz, S. et al. Monocytes/macrophages prevent healing defects and left ventricular thrombus formation after myocardial infarction. FASEB J. 27, 871-881 (2013).

4. Frangogiannis, N. G. et al. Critical role of monocyte chemoattractant protein$1 /$ CC chemokine ligand 2 in the pathogenesis of ischemic cardiomyopathy. Circulation 115, 584-592 (2007).

5. Morimoto, H. et al. Cardiac overexpression of monocyte chemoattractant protein-1 in transgenic mice prevents cardiac dysfunction and remodeling after myocardial infarction. Circ. Res. 99, 891-899 (2006).

6. Nahrendorf, M. et al. The healing myocardium sequentially mobilizes two monocyte subsets with divergent and complementary functions. J. Exp. Med. 204, 3037-3047 (2007).

7. Sunderkotter, C. et al. Subpopulations of mouse blood monocytes differ in maturation stage and inflammatory response. J. Immunol. 172, 4410-4417 (2004).

8. Yona, S. et al. Fate mapping reveals origins and dynamics of monocytes and tissue macrophages under homeostasis. Immunity 38, 79-91 (2013).

9. Varga, T. et al. Tissue LyC6-macrophages are generated in the absence of circulating LyC6- monocytes and Nur77 in a model of muscle regeneration. J. Immunol. 191, 5695-5701 (2013).

10. Hilgendorf, I. et al. Ly-6Chigh monocytes depend on Nr4al to balance both inflammatory and reparative phases in the infarcted myocardium. Circ. Res. 114, 1611-1622 (2014).

11. Panizzi, P. et al. Impaired infarct healing in atherosclerotic mice with Ly-6C(hi) monocytosis. J. Am. Coll. Cardiol. 55, 1629-1638 (2010).

12. Singla, D. K., Singla, R. D., Abdelli, L. S. \& Glass, C. Fibroblast growth factor-9 enhances M2 macrophage differentiation and attenuates adverse cardiac remodeling in the infarcted diabetic heart. PLOS ONE 10, e0120739 (2015).

13. Ben-Mordechai, T. et al. Targeting macrophage subsets for infarct repair. J. Cardiovasc. Pharmacol. Ther. 20, 36-51 (2015).

14. Kawahara, K., Hohjoh, H., Inazumi, T., Tsuchiya, S. \& Sugimoto, Y. Prostaglandin E2-induced inflammation: relevance of prostaglandin E receptors. Biochim. Biophys. Acta 1851, 414-421 (2015).

15. Wang, M. \& FitzGerald, G. A. Cardiovascular biology of microsomal prostaglandin E synthase-1. Trends Cardiovasc. Med. 20, 189-195 (2010).

16. Degousee, N. et al. Microsomal prostaglandin E2 synthase-1 deletion leads to adverse left ventricular remodeling after myocardial infarction. Circulation 117, 1701-1710 (2008).

17. Degousee, N. et al. Lack of microsomal prostaglandin E(2) synthase-1 in bone marrow-derived myeloid cells impairs left ventricular function and increases mortality after acute myocardial infarction. Circulation 125, 2904-2913 (2012).

18. Van Linthout, S., Miteva, K. \& Tschope, C. Crosstalk between fibroblasts and inflammatory cells. Cardiovasc. Res. 102, 258-269 (2014).

19. Lu, A. et al. EP3 receptor deficiency attenuates pulmonary hypertension through suppression of Rho/TGF-betal signaling. J. Clin. Invest. 125, 1228-1242 (2015).

20. Ingersoll, M. A. et al. Comparison of gene expression profiles between human and mouse monocyte subsets. Blood 115, e10-e19 (2010).

21. Kalinski, P. Regulation of immune responses by prostaglandin E2. J. Immunol. 188, 21-28 (2012)

22. Hui, Y. et al. Targeted deletions of cyclooxygenase- 2 and atherogenesis in mice. Circulation 121, 2654-2660 (2010).

23. Heusinkveld, M. et al. M2 macrophages induced by prostaglandin E2 and IL-6 from cervical carcinoma are switched to activated M1 macrophages by CD4+ Th1 cells. J. Immunol. 187, 1157-1165 (2011).

24. Weller, C. L. et al. Chemotactic action of prostaglandin E2 on mouse mast cells acting via the PGE2 receptor 3. Proc. Natl Acad. Sci. USA 104, 11712-11717 (2007).

25. Breyer, R. M., Bagdassarian, C. K., Myers, S. A. \& Breyer, M. D. Prostanoid receptors: subtypes and signaling. Annu. Rev. Pharmacol. Toxicol. 41, 661-690 (2001).

26. Zhang, J. et al. Cyclooxygenase-2-derived prostaglandin $\mathrm{E}(2)$ promotes injuryinduced vascular neointimal hyperplasia through the E-prostanoid 3 receptor. Circ. Res. 113, 104-114 (2013).

27. Landsman, L. et al. CX3CR1 is required for monocyte homeostasis and atherogenesis by promoting cell survival. Blood 113, 963-972 (2009).

28. Lionakis, M. S. et al. CX3CR1-dependent renal macrophage survival promotes Candida control and host survival. J. Clin. Invest. 123, 5035-5051 (2013).

29. Peng, X., Zhang, J., Xiao, Z., Dong, Y. \& Du, J. CX3CL1-CX3CR1 interaction increases the population of Ly6C-CX3CR1hi macrophages contributing to unilateral ureteral obstruction-induced fibrosis. J. Immunol. 195, 2797-2805 (2015).

30. Kaur, T., Hirose, K., Rubel, E. W. \& Warchol, M. E. Macrophage recruitment and epithelial repair following hair cell injury in the mouse utricle. Front. Cell. Neurosci. 9, 150 (2015).

31. Ishida, Y., Gao, J. L. \& Murphy, P. M. Chemokine receptor CX3CR1 mediates skin wound healing by promoting macrophage and fibroblast accumulation and function. J. Immunol. 180, 569-579 (2008).
32. Clover, A. J., Kumar, A. H. \& Caplice, N. M. Deficiency of CX3CR1 delays burn wound healing and is associated with reduced myeloid cell recruitment and decreased sub-dermal angiogenesis. Burns 37, 1386-1393 (2011).

33. Zhao, W., Lu, H., Wang, X., Ransohoff, R. M. \& Zhou, L. CX3CR1 deficiency delays acute skeletal muscle injury repair by impairing macrophage functions. FASEB J. 30, 380-393 (2015).

34. Jeon, S. H. et al. Mechanisms underlying TGF-betal-induced expression of VEGF and Flk-1 in mouse macrophages and their implications for angiogenesis. J. Leukoc. Biol. 81, 557-566 (2007).

35. Hohlfeld, T., Meyer-Kirchrath, J., Vogel, Y. C. \& Schror, K. Reduction of infarct size by selective stimulation of prostaglandin $\mathrm{EP}(3)$ receptors in the reperfused ischemic pig heart. J. Mol. Cell. Cardiol. 32, 285-296 (2000).

36. Zacharowski, K. et al. Selective activation of the prostanoid $\mathrm{EP}(3)$ receptor reduces myocardial infarct size in rodents. Arterioscler., Thromb, Vasc. Biol. 19, 2141-2147 (1999).

37. Zacharowski, K., Olbrich, A., Otto, M., Hafner, G. \& Thiemermann, C. Effects of the prostanoid EP3-receptor agonists M\&B 28767 and GR 63799X on infarct size caused by regional myocardial ischaemia in the anaesthetized rat. $\mathrm{Br}$. J. Pharmacol. 126, 849-858 (1999).

38. Martin, M. et al. Cardiospecific overexpression of the prostaglandin EP3 receptor attenuates ischemia-induced myocardial injury. Circulation 112, 400-406 (2005).

39. Sanchez-Alavez, M. et al. Night eating and obesity in the EP3R-deficient mouse. Proc. Natl Acad. Sci. USA 104, 3009-3014 (2007).

40. Ceddia, R. P. et al. The PGE2 EP3 receptor regulates diet-induced adiposity in male mice. Endocrinology 157, 220-232 (2016).

41. Yan, S. et al. Prostaglandin E2 promotes hepatic bile acid synthesis by an E prostanoid receptor 3-mediated hepatocyte nuclear receptor $4 \alpha /$ cholesterol $7 \alpha$-hydroxylase pathway in mice. Hepatology. doi: 10.1002/hep.28928 (2016).

42. Ricciotti, E. \& FitzGerald, G. A. Prostaglandins and inflammation. Arterioscler. Thromb, Vasc. Biol. 31, 986-1000 (2011).

43. Gross, S., Tilly, P., Hentsch, D., Vonesch, J. L. \& Fabre, J. E. Vascular wallproduced prostaglandin E2 exacerbates arterial thrombosis and atherothrombosis through platelet EP3 receptors. J. Exp. Med. 204, 311-320 (2007).

44. Lazarus, M. et al. EP3 prostaglandin receptors in the median preoptic nucleus are critical for fever responses. Nat. Neurosci. 10, 1131-1133 (2007).

45. Stapel, B. et al. Low STAT3 expression sensitizes to toxic effects of betaadrenergic receptor stimulation in peripartum cardiomyopathy. Eur. Heart J. 38, 349-361 (2016)

46. Lang, R., Rutschman, R. L., Greaves, D. R. \& Murray, P. J. Autocrine deactivation of macrophages in transgenic mice constitutively overexpressing IL-10 under control of the human CD68 promoter. J. Immunol. 168, 3402-3411 (2002).

47. Kumar, D. et al. Distinct mouse coronary anatomy and myocardial infarction consequent to ligation. Coron. Artery Dis. 16, 41-44 (2005).

48. Ma, X. L. et al. Inhibition of p38 mitogen-activated protein kinase decreases cardiomyocyte apoptosis and improves cardiac function after myocardial ischemia and reperfusion. Circulation 99, 1685-1691 (1999).

49. Matsushita, K. et al. Interleukin-6/soluble interleukin-6 receptor complex reduces infarct size via inhibiting myocardial apoptosis. Lab. Invest. 85, 1210-1223 (2005).

50. Liang, H. et al. Increased expression of pigment epithelium-derived factor in aged mesenchymal stem cells impairs their therapeutic efficacy for attenuating myocardial infarction injury. Eur. Heart J. 34, 1681-1690 (2013).

51. Horie, S. et al. CD200R signaling inhibits pro-angiogenic gene expression by macrophages and suppresses choroidal neovascularization. Sci. Rep. 3, 3072 (2013).

52. Shi, M. et al. Myeloid-derived suppressor cell function is diminished in aspirintriggered allergic airway hyperresponsiveness in mice. J. Allergy Clin. Immunol. 134, 1163-1174 e1116 (2014).

53. Yan, X. et al. Temporal dynamics of cardiac immune cell accumulation following acute myocardial infarction. J. Mol. Cell. Cardiol. 62, 24-35 (2013).

54. Hannenhalli, S. et al. Transcriptional genomics associates FOX transcription factors with human heart failure. Circulation 114, 1269-1276 (2006).

55. Roukens, M. G. et al. Control of endothelial sprouting by a Tel-CtBP complex. Nat. Cell Biol. 12, 933-942 (2010).

56. Kurrelmeyer, K. M. et al. Endogenous tumor necrosis factor protects the adult cardiac myocyte against ischemic-induced apoptosis in a murine model of acute myocardial infarction. Proc. Natl Acad. Sci. USA 97, 5456-5461 (2000).

57. Abdel-Latif, D. et al. Rac2 is critical for neutrophil primary granule exocytosis. Blood 104, 832-839 (2004).

\section{Acknowledgements}

This work was supported by Grants from the National Natural Science Foundation of China (81525004, 91439204, 91639302, 31200860, 81400239 and 31371154), the Ministry of Science and Technology of China (2012CB945100), Shanghai Committee of Science and Technology (14JC1407400, 15140902000). Ying Yu is a fellow at the Jiangsu Collaborative Innovation Center for Cardiovascular Disease Translational Medicine. 


\section{Author contributions}

J.T., G.C.,: performed statistical analysis; J.W., M.L., Ying Yu: handled funding and supervision; J.T., Y.S., G.C., K.W., Q.W., J.Z., J.Q., G.L., S.Z., Yu Yu, J.W., M.L.: acquired the data; J.T., Ying Yu: conceived and designed the research; J.T., Ying Yu: drafted the manuscript; M.L., Ying Yu: made critical revision of the manuscript for key intellectual content.

\section{Additional information}

Supplementary Information accompanies this paper at http://www.nature.com/ naturecommunications

Competing financial interests: The authors declare no competing financial interests.

Reprints and permission information is available online at http://npg.nature.com/ reprintsandpermissions/
How to cite this article: Tang, J. et al. Activation of E-prostanoid 3 receptor in macrophages facilitates cardiac healing after myocardial infarction. Nat. Commun. 8, 14656 doi: $10.1038 /$ ncomms14656 (2017)

Publisher's note: Springer Nature remains neutral with regard to jurisdictional claims in published maps and institutional affiliations.

(c) (i) This work is licensed under a Creative Commons Attribution 4.0 International License. The images or other third party material in this article are included in the article's Creative Commons license, unless indicated otherwise in the credit line; if the material is not included under the Creative Commons license, users will need to obtain permission from the license holder to reproduce the material To view a copy of this license, visit http://creativecommons.org/licenses/by/4.0/

(C) The Author(s) 2017 